\title{
Zur Analysis der Schwerkraftswirkung auf die Entwicklung des Froscheies.
}

\author{
Von
}

Dr. med. Max Mosrkowski.

Hierzu Tafel XIX und ein Schema im Text.

In meiner Arbeit über den Einfluss der Schwerkraft etc. (37) hatte ich rein descriptiv festgestellt, dass die Schwerkraft insofern als eine für die Entwicklung des Froscheies notwendige, gestaltende Ursache zu bezeichnen ist, als sie dem $\mathrm{Ei}$ eine Symmetrieebene schafft und so die Medianebene des künftigen Embryos bestimmt. Es handelt sich jetzt darum, diese Wirkungsweise der Schwerkraft einer tieferen, causal-analytischen Betrachtung zu unterziehen. Die Frage lautet, welcher Categorie von Reizen ist die Wirkung der Schwerkraft auf die Entwicklung des Froscheies zu subsummieren? Handelt es sich, wie O. Sch u l t ze meint, um einen die gesamte Zellteilung und Organbildung des Froscheies beherrschenden Reiz $(47,48)$, oder um einen ontomorphogenen Reiz im Sinne von Herbst (23, pag. 822) und zwar um welchen: um einen nur lokalisierenden, um einen „Auslösungsreiz", einen "Umschaltungsreiz", einen "struckturellen" Reiz, oder liegt etwa gar nur eine einfache hydrostatische Erscheinung vor, bedingt durch die ordnende Wirkung der Schwerkraft auf die verschieden schweren Substanzen des Eidotters?

Nun hat meine Auffassung über dië Rolle, welche die Schwerkraft in der Ontogenese des Froscheies spielt, auf verschiedenen Seiten lebhaften Widerspruch hervorgerufen. Kaum zwei Monate nach der Veröffentlichung meiner Arbeit sind schon drei Aufsätze erschienen, welche meine Ansicht $z u$ widerlegen suchen, zwei sachliche von Morgan (56) und Kathariner (54) und eine von Roux, (57) die in dem Tone gehalten ist, der aus den Polemiken dieses Autors mit M. Verworn, O. Hertwig, O. Schultze, Hans Driesch hinlänglich bekannt ist. Mein hochverehrter Lehrer Prof. Keibel (55) hat bereits Gelegenheit genommen auf die Angriffe Roux's zu antworten und die zahlreichen Irrtüner $z u$ berichtigen, die Roux beim Zitieren eigner und 
fremder Arbeiten in der Eile unterlaufen sind. Ich bin daher in der angenehmen Lage auf jede weitere persönliche Polemik verzichten und mich ausschliesslich den rein sachlichen Einwänden meiner Gegner widmen zu können.

In erster Linie kann ich zu meiner Genugthuung constatieren, dass sowohl Morgan, wie Kathariner, wie Roux nicht bestreiten, dass unter gewissen Bedingungen die Medianebene des künftigen Embryos durch die Schwerkraft bestimmt werden kann: "It should be carefully noted that I do not claim that gravity may not under certain conditions produce this result" (56, pag. 314). „Dass die Schwerkraft in der von ihm beschriebenen Weise beim Zustandekommen der bilateralen Symmetrie beteiligt sein kann, will ich keineswegs bestreiten" (54, pag. 292). "Die Bestimmungen 2 u. 4." (i. e. Bestimmung der Medianebene und Lokalisation des Embryos) „können auch durch entsprechende künstlich veranlasste Anordnung der Dottersubstanz (durch schiefe Zwangslage) bewirkt werden!: In der That giebt es ja auch für die Möglichkeit der Bestimmung der Medianebene durch Schwerkraftswirkung unwiderlegliche Beweise:

1. Born (2) fand bei seinen Versuchen mit in schiefer Zwangslage fixierten Eiern, dass zwar die erste Furche nicht immer, wohl aber die Medianebene stets durch den Strömungsmeridian, i. e. die durch die Schwerkraft geschaffene Symmetrieebene ging.

2. O. Schultze (45a) gelang es durch Umdrehen auf dem Zweizellenstadium durch die Schwerkraftswirkung derartige Umlagerungen im. Froschei hervorzurufen, dass statt eines Embryos zwei aus einem. Ei entstanden. Desgleichen ist es Morgan-geglückt aus einem auf dem Zweizellenstadium angestochenen Ei einen Ganzembryo zu züchten, wenn er das Ei nach dem Anstich mit dem weissen Pol nach oben orientierte, während aus angestochenen Eiern, die mit dem weissen Pol nach unten gerichtet waren, ein Halbembryo entstand (34a). Diese beiden Fälle sind besonders beweisend dafür, dass durch Schwerkraftswirkung die Medianebene des Embryos bestimmt werden kann, weil es sich hier offenbar um eine reine Schwerkraftswirkung handelt und die von $\mathrm{Roux}$ behauptete Bestimmung der Medianebene durch die Copulationsrichtung der Vorkerne bei 
diesen, erst auf dem Zweizellenstadium gedrehten Eiern ganz sicher ausgeschlossen werden muss.

Ich bin also berechtigt folgenden Satz als sicher feststehend auszusprechen: Die Medianebenedes künftigen Embryos kann durch Schwerkraftswirkung bestimmt werden. Mit der Konstatierung dieser von Roux ausdrücklich zugegebenen Thatsache scheint mir aber die Auffassung dieses Autors dass die Medianebene unter normalen Verhaltnissen durch die Copulationsrichtung der Vorkerne bestimmt wird, bereits widerlegt zu sein, und zwar aus folgenden Gründen: Wenn man mit Roux annimmt, dass das eindringende Spermatozoon die Fähigkeit besitze das Dottermaterial so umzuordnen, dass aus dem bis dahin radiär gebauten, nach allen Richtungen isotropen Ei ein bilateral-symmetrisches, anisotropes Gebilde wird, so liegt hier offenbar ein sehr compliciertes Geschehen vor, dem ein sehr feiner bis ins Einzelne specialisierter Mechanismus zu Grunde liegen müsste. Ja man muss eigentlich in dieser Thitigkeit des Spermatozoons den Dotter so umzuorden, dass „zum richigen Idioplasson des Kernes das richtige Protoplasma kommt" - um emen Ausspruch von Roux umzudrehen - einen specifisch vitalen Vorgang erblicken, obwohl Roux diesen Ausdruck sorgfïltig vermeidet. Ebenso muss die rahigkeit des Protoplasmas drehend auf die Kerıspindel zu wirken, damit in den Fallen, wo durch die Schwere - bei schiefer Zwangslage - eine symmetrische Anordnung des Dotters geschaffen ist gleich qualifiziertes Material in Zellleib und Zellkern auf derselben Seite zu liegen kommen, eine typisch vitale Eigenschaft erblickt werden. Im Falle der Umordnung des Dotters aber durch die Schwerkraft liegt eine einfache hydrostatische Erscheinung vor, durch welche zwar eine Ungleichheit in der Verteilung des Dotters, aber doch nimmermehr eine richtige qualitative Materialscheidung bewirkt werden kann. Eins von beiden kann nur der Fall sein. Entweder handelt es sich um ein ausserordentlich compliziertes Geschehen, das nach der Definition des Wortes als exquisit vitales bezeichnet werden muss, oder um einen rein mechanischen Vorgang. Es ist unmöglich, dass dieser für jenes gleichsam vikarierend eintreten kann. Es ist ja auch von Roux für seine Behauptung, dass die Medianebene des Embryo durch die Copulationsrichtung der Vorkerne bestimmt würde nicht ein einziger Einwands freier 
Beweis erbracht worden. Seine Versuche mit lokalisierter Befruchtung sind derartig, dass ich sie mit viel mehr Recht als Stütze für meine Auffassung ansehen kann (vergl. 55). Die Eier wurden (44, pag. 359) ohne Wasserzusatz gerade aufgesetzt und von einer Seite her, nach Anschneiden der Gallerthülle, befruchtet. Es ist klar, dass die Eier sich nach der Seite neigen mussten: von welcher der Samen zugesetzt wurde (es ist ja allgemein bekannt, dass die Eier sich immer nach der Seite des Wasserzutritts neigen). Da nun die Eier erst 30 Minuten nach der Besamung mit reichlichem Wasser versehen wurden, also im Ganzen mindestens eine Stunde in schiefer Zwangslage waren, so musste sich all ihnen eine Symmetrieebene durch Schwerkraftswirkung ausbilden, die bei der ganzen Versuchsanordnung mit dem Befruchtungsmeridian übereinstimmte. Só oft Roux versuchte, schräg zur Symmetrieebene der Einstellung zu befruchten, hatte er eingestandenermaassen eclatante Misserfolge (44, pag. 399-403). Es berührt merkwürdig, dass Roux, der bei seinen lokalisierten Befruchtungsversuchen die Eier so lange in Zwangslage hielt, und der schon selbst angegeben hat (44, pag. 968), dass die Eier auch normaler Weise sich $30-45$ Minuten nach der Besamung in Zwangslage befinden, - es berülırt merkwürdig, dass derselbe Autor denjenigen Forschern, welche die von ihm postulierte Uebereinstimmung der Pigmentstrasse mit der Ebene der ersten Furche nicht bestätigen konnten, vorwirft, sie hatten ihre Eier in Zwangslage gehalten. (Gegen van Bambecke, pag. 369, und gegen R. Fick, pag. 376) [vergl. 55]. Mit dem Nachweis, dass bei Roux's Fundamentalversuchen, auf welchen seine ganze Theorie basiert, die Medianebene durch die Schwere bestimmt worden ist, schwebt Roux's Meinung von der Bestimmung der Medianebene durch die Kopulationsrichtıng völlig in der Luft.

In zweiter Linie landelt es sich um Beantwortung der Frage, ob unter normalen Verhälnissen die Medianebene gleichfalls durch Schwerkraftswirkung bestimmt wird. Einige Zeit nach der Befruchtung wird die weisse Hemisphäre der Eier von R. fusca durch einen Halbmond von grauer Farbe vergrössert, wie übereinstimmend von $\operatorname{Roux}(44$, p. 355), O. Schultze $(45,47,48,49)$ und Morgan (32) berichtet wird und wovon sich zum Uebertluss jedermann leicht überzengen kann. Auf 
diese Weise erhält das bis dahin radiär gebaute $\mathrm{Ei}$ eine Symmetrieebene und wird bilateral symmetrisch. Diese Symmetrieebene wird nun, wie ich weiter unten erweisen werde (ich befinde mich hierbei in völliger Uebereinstimmung mit Ro a x [44], O. Schultze $[45,47,48,49]$, O. Hertwig [26, 27], und Morgan und Tsuda [32]) zur Medianebene des Embryos. Gelingt es also zu zeigen, dass das graue Feld durch Schwerkraftswirkung entsteht, so ist die oben aufgeworfene Frage in positivem Sinne erledigt.

Kurz nach der Besamung befinden sich die Eier, wie ich in meiner vorigen Arbeit des Naheren ausgeführt habe in physiologischer Zwangslage. ${ }^{1}$ ) Diese Zwangslage ist bei der ungeheuren Mehrzahl der Eier eine schiefe. Die Wahrscheinlichkeit nämlich, dass ein Ei bei der Eiablage genau ,normal" i. e. mit dem weissen Pol genau nach unten zu liegen kommt, ist wie $1 \mathrm{zu} \infty$. Ich verstehe nicht recht, was $K$ a thariner an dieser Behauptung auszusetzen hat. Gewiss ist die Wahrscheinlichkeit, dass das Ei genau lotrecht orientiert ist, durchaus keine geringere als die jeder anderen Lage. Da aber das Ei als kugelförmiger Körỵer unendlich viele Achsen besitzt, so ist die Wahrscheinlichkeit, dass es gerade in eine ganz bestimmte Achse fällt wie $1: \infty$ (vergl. Keibel 55). Davon kann vollends keine Rede sein, dass der exzentrische Schwerpunkt des Eies grade die Stellung mit lotrechter primärer Achse begünstigen könnte, da ja die Eier nicht einzeln sondern in ganzen Ballen abgelegt werden, die Lage ihres eigenen Schwerpunktes also gar nicht in Betracht kommt. Ich kann also durchaus nicht zugeben, dass aus meiner Aufstellung Consequenzen folgen, „deren Unnatürlichkeit allein letztere schon erschüttern dürfte" $(54$, pag. 293). Nun werden sich natürlich auch die Eier, deren primäre Achse von der vertikalen Stellung nur um wenige Grade abweicht, hinsichtlich der Erhaltung einer Symmetrieebene in ungünstiger Lage be-

1) Roux hat freilich schon im Jahre 1894 (pag. 968) darauf hingewiesen, dass die Eier sich die ersten 30-45 Minuten nach der Besamung in Zwangslage befinden. Die daraus folgenden Consequenzen, dass nämlich die Eier dann unter denselben Bedingungen sich befinden, wie Borns Zwangslageneier, also dieselben Umordnungen in ihnen zu Stande kommen müssen, hat er aber nicht gezogen. Anf diese Consequenzen habe, meines Wissens, ich, als erster, hingewiesen. 
finden. Es ist ja aber allen Experimentatoren bekannt, dass von einem Laichballen, sowohl bei künstlicher Befruchtung als auch in der Natur stets eine Anzahl Eier unentwickelt bleibt, respektive während der ersten Furchungsstadien abstirbt. Jedenfalls ergiebt eine einfache Ueberlegung, dass der bei Weitem grösste Teil der Eier bei der Ablage nicht genau oder annähernd senkrecht, sondern mit schiefer primärer Achse orientiert werden muss. Bei Eiern aber, die sich eine halbe Stunde oder länger in schiefer Zwangslage befinden, sinkt, wie wir aus den Versuchen von, Born (2) wissen, der weisse Dotter, an der Stelle wo er am höchsten steht, längs der Peripherie ab unter Hinterlassung einer weissen peripheren Platte, die vom aufsteigenden braunen Dotter unterlagert wird. Diese Stelle hat äusserlich die charakteristische graue Farbe. Da nun, wie ich noch einmal ausdrücklich betone, eben befruchtete Eier sich auch normaler Weise unter denselben Bedingungen befinden wie künstlich in Zwangslage gehaltene, so haben wir nicht die geringste Veranlassung das normaler Weise auftretende graue Feld anders zn deuten als das, welches in Borns Experimenten auftrat. Nun ist von Morgan die Frage aufgeworfen worden, ob nicht vielleicht das graue Feld sich an einer präformierten Stelle entwickelt und das $\mathrm{Ei}$ dann nach der Befruchtung sich so dreht, dass diese Stelle den höchsten Punkt der weissen Hemisphäre einnimmt? (Does the grey crescent develop in a pre-organized part of the egg, and if so, does the egg rotate after fertilization so that this part turns uppermost?" (56, pag. 315). Demgegenuiber ist folgendes zu erwidern:

1. Das graue Feld erscheint auch bei unbefruchteten Eiern, an der Stelle, wo der weisse Dotter am höchsten heraufreicht, allerdings viel später. Morgan hat das selbst beobachtet: „The latter appears, however, in the unfertilized egg after 24 hours ${ }^{1}$ ), if the eggs are allowed to stand quietly. The grey area appears on that part of the egg that stands uppermost. Usually it appears in the region between the black and the white area, but $I$ have seen it appear even at the white pole if this happened to be the highest point of the egg" (pag. 315).

1) Bei R. fusca erscheint das graue Feld am unbefruchteten Ei schon nach 5-6 Stunden. 
Hier kann doch wohl von einer durch die Befruchtung präformierten Stelle nicht gesprochen werden. Grade das von Morgan beobachtete Erscheinen des grauen Feldes direkt am weissen Pol in den Fällen, dass derselbe direkt nach oben gerichtet war, spricht ganz besonders dafür, dass das graue Feld durch Schwerkraftswirkung entsteht. Wir wissen durch Born, dass in diesem Fall der weisse Dotter nicht längs eines Meridians, sondern nach allen Seiten hin abfliesst. Der braune Dotter steigt dann in der Mitte des Eies auf und unterlagert die auf der Höhe des weissen Poles stehen gebliebene weisse Platte, wodurch das graue Feld am weissen Pole in Erscheinung tritt. Figur 22 von Born, die ich auf pag. 37 meiner Arbeit (37) reproduziert habe, zeigt wie ein solches Ei im Durchschnitt aussieht.

2. Bei in schiefer Zwangslage fixierten Eiern erscheint das graue Feld nach etwa dreiviertel Stunden, also fast zur selben Zeit, wie normal und ist dann auch ebenso gross wie das normale graue Feld, vergrössert sich dann aber allmählig in der Richtung der Schwerkraftswirkung. Ein Rotieren des Eies ist bei diesen in vollkommener Zwangslage befindlichen Eiern natürlich unmöglich. Wir sind also abermals berechtigt Folgendes zu bebaupten: I)as normaler Weise auftretende graue Feld entsteht durch Schwerkraftswirkung.

Die dritte Frage lautet: Besitzen alle Eier ein graues Feld? Morgan giebt an, dass er in manchen Bruten ein graues Feld nicht habe finden können. Ich kann das für $R$. fusca bestâtigen. Jedoch habe ich jedesmal schon vor der Befruchtung gesehen, ob sich bei den Eiern der betreffenden Brut ein graues Feld entwickeln wird, oder nicht. Bei Eiern nämlich, die besonders stark pigmentiert sind, tritt infolge der dann sehr dicken Pigmentrinde das graue Feld ausserlich nicht in Erscheinung. Jedenfalls ist auch die Zahl der Bruten, bei denen es infolge der sehr starken Pigmentierung zu einer ausserlichen Entwicklung des grauen Feldes nicht kommt, verhältnismässig sehr klein gegen die, bei denen ein wohl ausgebildetes graues Feld in Erscheinung tritt. Da nun das Fehlen des grauen Feldes bei den stark pigmentierten Eiern sich sehr einfach erklärt, so scheint mir auch unsere dritte Frage in positivem Sinne beantwortet werden zu müssen: Die normale Entwicklung der Eier 
von R. fuscavollzieht sich unter Bildung eines grauen Feldes. Mit anderen Worten: Unter normalen Verhälnissen wird die Medianebene des Embryos von R. fusca durch die Schwerkraft bestimmt. Ich glaube nicht, dass sich angesichts des von mir erbrachten Thatsachenmaterials gegen diese These irgend etwas einwenden lässt.

Wir kommen nun zu der vierten Frage. Dieselbe lautet: Kann sich das Froschei auch bei Fehlen jeglicher Schwerkraftswirkung entwickeln? Oder anders formuliert: Sind im befruchteten Froschei Kräfte vorhanden, die ihm auch bei Fehlen der Schwerkraftswirkung eine Medianebene schaffen können? Der erste, der das Nichtnötigsein der Schwerkraft für die Entwicklung des Froscheies experimentel zu beweisen suchte, war Roux (44, pag. 256-276). Seine Versuche sind aber, abgeseben von allen anderen Einwänden, schon aus dem Grunde nicht stichhaltig, weil er die Eier stets erst eine halbe Stunde nach der Besamung dem Eintluss der Schwerkraft zu entziehen versuchte, also zu einer Zeit, wo die Arbeit der Schwerkraft schon gethan war. Dasselbe gilt von den ersten Versuchen Kathariners. Angeregt durch meine Ausfiihrungen haben nun Morgan (56) und $K$ athariner (54) während der diesjährigen Laichperiode versucht, die Eier dem Einfluss der Schwerkraft zu entziehen bevor dieselbe ihre gestaltende Wirksamkeit, i. e. die Anordnung des Eimaterials symmetrisch zu nur einer Symmetrieebene, auszuïben vermochte. Ob der von Kathariner ersonnene Apparat in der Tat geeiguet ist, die richtende Wirksamkeit der Schwerkraft auszuschalten, lasse ich dahingestellt. Aber selbst wenn er es ist, so ist $\mathrm{K}$ ath a riners Beweis darum nicht minder misslungen. Denn da ich nicht, wie O. Schultze, glaube, dass die Schwerkraft bestandig die Zellteilung und Organbildung beherrscht, so vertrugt es sich vollkommen mit meinen Anschauungen, dass Eier, „die niemals solange in abnormer Lage bleiben konnten, dass ihre Massen Zeit gehabt hätten sich gegeneinander zu verschieben" (37, pag. 44) sich normal entwickelten, wenn nur, bevor sie dem Einfluss der Schwerkraft entzogen wurden, die von der Schwerkraft veranlasste symmetrische Verteilung des Dotters schon erfolgt war. Nun hat Kathariner bei seinen diesjährigen Versuchen dic Eier im Minimum 7 Minuten nach der Befruchtung 
auf seinen Apparat gebracht. Auch diese Eier entwickelten sich normal und damit glaubt Kathariner das Nichtnötigsein der Schwerkraft für die Entwicklung des Froscheies definitiv nachgewiesen zu haben. Icb kann ihm hierin durchaus nicht zustimmen. Freilich das aussere Zeichen für die im Innern des Eies stattfindenden Dotterumlagerungen (das graue Feld) erscheint im Mittel erst eine halbe bis dreiviertel Stunden nach der Besamung, aber Born hat schon in seiner Arbeit darauf hingewiesen (2, pag. 295), dass man auf Schnitten innere Umlagerungen schon nachweisen kann, zu einer Zeit, wo von aussen noch nicht das Geringste sichtbar ist. Und es ist doch selbstverständlich, dass diese inneren Umlagerungen noch früher beginnen als sie nachzuweisen sind. Bei meiner Auffassung von der Wirksamkeit der Schwerkraft, die ich in vorliegender Arbeit näher austïhren werde, handelt es sich nur darum, dass durch Schwerkraftswirkung der ursprünglich radiare Bau des Froscheies zerstört und durch einen bilateral-symmetrischen ersetzt wird. Das radiär gebaute $\mathrm{Ei}$ ist isotrop. Der Embryo kann sich auf jedem Meridian entwickeln, wie ans Borns Versuchen mit in-schiefer Zwangslage fixierten Eiern und aus Roux's lokalisierten Befruchtungsversuchen hervorgeht, das bilateral-symmetrische $\mathrm{Ei}$ ist anisotrop, der Embryo kaun sich nur anf dem Meridian der Symmetrieebene und sogar nur auf einer ganz bestimmten Stelle dieses Meridians entwickeln. Es liegt auf der Hand, dass die Verschiebungen der beiden Dotterarten gegen einander bei der grossen Kleimheit des Froscheies relativ gar nicht bedeutend zu sein brauchen, um dieses Resultat, Zerstörung des radiären Baues des Eies, hervolzurufen. Dafür aber, dass derartige Verschiebungen des Dotters während der 7 Minuten, in denen das Ei im Minimum der Wirkung der Schwerkraft ausgesetzt war, n i ch t erfolgt sind, hat K a th a riner keine Beweise erbracht. Ich weiss daher nicht, woher er die Berechtigung nimmt zu sagen, "dass die Eier zu einer Zeit unter jene Bedingungen kamen, wo sie noch die ihnen angeborene Struktur hatten:. Ich könnte mit dem gleichen Rechte behaupten, dass durch Kathariners Versuche grade bewiesen ist, dass die Eier nicht mehr die ihnen angeborene Struktur hatten. Die Versuche Kathariners zeigen, dass ich Unrecht hatte, als ich in der Ausscheidung des Perivitellins den einzigen 
Faktor erblickte, durch welchen die Abnahme der Cohäsion des Dotters nach der Befruchtung bedingt wird. Es ist dies sicherlich ein Faktor, wie aus dem Verhalten unbefruchteter Eier hervorgeht, aber eben nur einer. Dies wird einmal durch die Kathariner'schen Versuche bewiesen, dann aber hauptsächlich durch die Ueberlegung, dass Zwangslageneier, bei denen doch nur sehr wenig Perivitellin austreten kann, in beinahe der gleichen Zeit auf den Einfluss der Schwerkraft reagieren, wie normale Eier, bei denen das Perivitellin ganz austreten kann. Ich ziehe daher einen Einwand, den ich Born gegenüber erhoben habe, in gewisser Weise zurück. Born spricht von einer consistenzverändernden Wirkung des Spermatozoon. Eine solche liegt nun meiner Ansicht nach nicht vor. Wobl aber halte ich es für denkbar, dass am unbefruchteten Ei der Zellinhalt fester an der Pigmentrinde haftet, als am befruchteten, und dass diese Lockerung durch eine chemische Beeintlussung von Seiten des Spermatozoon im Momente der Berührung mit der Eirinde erfolgt. Derartige chemische Beeinflussung des Spermatozoen im Moment der Berührung mit dem Ei sind ja uns langst vertraute Erscheinungen. Ich erinnere an die Bildung des Empfängnishügels bei Seeigeleiern, an die Entstehung der Dotterhaut, an die von Born entdeckte Tatsache, der Dellenbildung, die an Eiern von R. esculenta eine halbe Stunde nach der Besamung mit Samen von R. fusca auftritt (2a), sowie an die Frörterungen die Born an diesen Befund knüpft. Ob, wie dieser Autor und nach ihm Roux (44, pag. 293/295) behaupten, der Unterschied der beiden Dotterarten im spezitischen Gewicht unmittelbar nach der Befruchtung (also infolge der unmittelbaren Contaktwirkung des Spermatozoon) wirklich grösser wird wie vorher, kann ich nicht entscheiden. Hierin aber stimme ich mit diesen Forschern überein, dass im Momente der Berührung des Samens mit dem Ei Veränderungen wahrscheinlich chemischer Art in demselben vor sich gehen, welche der Schwerkraft das Uebergewicht über die Cohäsion des Dotters verschaffen. Roux behauptet selber, dass das Ei seine definitive Stellung schon 15 Minuten nach der Befruchtung einnimmt, allerdings ant Grund von Versuchen, deren Unzulanglichkeit Keibel (55) in seiner Entgegnung im anat. Anzeiger ins hellste Licht gesetzt hat. Aber gesetzt; diese Behanptung Roux's wäre besser 
fundiert als sie es ist, so wäre damit klipp und klar bewiesen, dass die Medianebene des Embryos jedenfalls viel früher normiert wird, als die Pigmentstrasse des Spermatozoon entsteht. denn die Symmetrieebene der Einstellung wird ja, wie Roux, wenigstens für R. esculenta, selber angiebt, zur Medianebene des Embryos. Es sind also auch die neuen Versuche Kathariners nicht im Stande zu beweisen, das eventuell die Medianebene des Embryos auch durch innere Krafte bei Ausschluss der Schwere entstehen kann, Kathariner hat eben die Schwerkraft gar nicht vom Moment ihrer Wirksamkeit an ausgeschlossen, sondern erst zu einer Zeit, wo innere Umordnungen des Eiinhaltes durch Schwerewirkung schon haben erfolgen können. Noch weniger ist der von Morgan angestellte Versuch (56) im Stande dies zu beweisen. Morgan liess die Eier einer Kröte nach der Befruchtung eine halbe Stunde lang von einem seiner Assistenten herumbewegen, in welcher Weise wird nicht năher angegeben. "The toad was killed and the long strings of eggs were removed from the uterus and put at once into a small jar of water which was kept constantly whirling around by my assistant. Sperm was added and for half an hour the eggs were kept constantly turning over and over with great irregularity and with considerable rapidity" (56, pag. 314). Es ist im höchsten Maasse zweifelhaft und zum Mindesten gänzlich unbewiesen, dass bei dieser Versuchsanordnung das gewünschte Resultat auch wirklich erreicht wird, nümlich dass der Effekt, den eine richtende Kraft in einem Momente setzen will im nächsten Moment durch eine in anderer Richtung wirkende Kraft wieder aufgehoben wird. Eine halbe Stunde später brachte Morgan die Eier auf einen Apparat, in welchem sie durch einen Wasselstrom in bestandige, ungeordnete Bewegung versetzt wurden, hier wird der gewollte Effekt eventuell erreicht, dafür aber, dass die Eier sich auch in der ersten halben Stunde nach der Besamung unter solchen die Wirkung der Schwere ausschliessenden Bedingungen befunden haben, vermisse ich einen strikten Beweis. Die vierte Frage also, ob das $\mathrm{Ei}$ sich unter Ausschluss der Schwere entwickeln kann, ist meines Erachtenis noch ungelöst. Zahlreiche systematisch angestellte Versuche werden darüber Autschluss geben müssen. Freilich scheint es mir höchst unwahrscheinlich. dass eine normale Ent- 
wicklung des Froscheies bei Ausschluss der Schwere erfolgen kann, da ja das Froschei seinem ganzen Bau nach der Einwirkung der Schwerkraft angepasst erscheint, und ich einwandfrei bewiesen zu laben glaube, dass unter normalen Verhältnissen die Medianebene des Embryo durch die Schwere bestimmt wird. Damit erledigen sich auch die drei Punkte, die Morgan am Schluss seiner Arbeit als zu ermitteln hinstellt: "The critical points that now remain to be determined are: First, does the point of entrance of the Spermatozoon determine the bilaterality of the egg, as claimed by Roux; or Second, does the grey crescent develop in a pre-organized part of the egg and if so does the egg rotate after fertilization so that this part turns uppermost; or, Third, does the grey crescent appear at any point on the egg that happens to lie uppermost?" Ich habe erstens gezeigt, dass von einer Bestimmung der Medianebene durch die Copulationsrichtung der Yorkerne keine Rede sein kann, da Roux für diese Behauptung so $\mathrm{kümmerliche}$ B ew eise erbracht hat, dass sie völlig in der Luft schwebt. Nur viermal von fünfzehn Fällen ist es ihm gelungen erste Furche und Pigmentstrasse durch den ron ilum schräg z u l Symmetrieebene der Einstellung gewahlten Befruchtungsmeridian gehen zu lassen (?); in allen übrigen Fallen ist bei Roux's lokalisierten Befruchtungsversuchen die Versuchsanordnung, wie ich oben gezeigt habe, delartig, dass nicht durch die Befruchtungsrichtung, sondern durch die Schwere die Medianebene bestimmt wurde. Ich habe zweitens gezeigt, dass das graue Feld nicht an präformierter Stelle entsteht, sondern an der Stelle, wo das weisse Feld am höchsten heraufragt. Beweis: B orns Versuche an in schiefer Zwanglage fixielten Eiern, bei denen eine nachtrugliche Rotation des Eies ausgeschlossen ist, sowie der Umstand, dass auch bei unbefruchteten Eiern an der Stelle, wo das weisse Feld am höchsten steht, ein graues Feld erscheint. Hier kann doch wohl von einer durch die Befruchtung präformierten Stelle nicht die Rede sein. Damit ist auch Punkt drei erledigt.

Nachdem so der Einfluss der Schwere auf die Entwicklung des Froscheies, für das normale Verhalten wenigstens, sicher erwiesen ist, kommen wir dazu diese Wirksamkeit der Schwerkraft näher zu analysieren. 
Der erste, der dieses Problem in Angriff genommen hat, Pflüge r, hat, wie Herbst ganz richtig bemerkt (23, pag. 735, 24, pag. 28), die Frage nicht scharf und präzis genug gestellt und kommt demgemäss auch zu keiner ganz klaren Beantwortung derselben. Er stellte sich vor, dass sowohl die gesamte Zellteilung als auch die spatere Organbildung dauernd nach einem uubekannten Gesetz von der Schwerkraft "beherrscht" würden. Es lässt sich, wie auch Herbst meint, herauslesen, dass er sich wohl die betreffenden Entwicklungsprozesse als Barymorphosen, wie wir heute sagen würden, gedacht hat $(40,41,42)$. Pflüger hatte ferner angenommen, dass normaler Weise kausale Beziehungen zwischen den Richtungen der ersten Furchen und den späteren Achsen des Embryos bestünden. Roux hat fast zur selben Zeit die gleiche Behauptung aufgestellt. Doch .. hat sich diese Meinung in der Folgezeit als irrtümlich erwiesen. Es wird überhaupt, um Verwirrungen zu vermeiden, gut sein, scharf zwischen Wirkung der Schwerkraft anf die Furchung und Wirkung der Schwerkraft auf die Organbildung zu unterscheiden.

\section{Kapitel.}

\section{Einfluss der Schwerkraft auf dio Zellteilung.}

Was den Einfluss der Schwerkraft auf die Zellteilung betriffit, so hat schon Born durch seine fundamentalen Untersuchungen an in sehiefer Zwangslage fixierten Eiern nachgewiesen, dass ein direckt richtender Einfluss der Schwerkraft auf die 'Teilung nicht besteht, sondern nur ein indirekter. Bei schiefer Zwangslage wird der Nahrungsdotter unten und der Bildungsdotter oben angesammelt. Die Kerne folgen dem Bildungsdotter, vermutlich weil zwischen Kern und Bildungsdotter feste Beziehungen bestehen. Hier liegt zweifellos nichts weiter als eine einfache hydrostatische Erscheinung vor. „Die Schwerkraft wirkt ordnend auf die verschieden spezifisch schweren Substanzen des dickflüssigen Eiinhalts; das ist alles." (24, pag. 29.)

Diesem Urteil scheinen nun aber - worauf Herbst meiner Meinung nach viel zu wenig Gewicht legt - die thatsilchlich von Pflüger gemachten Befunde zu widersprechen. Pfll üger fand, dass stets die obere Hemisphäre in $\mathrm{kle}$ inere, die untere in grössere Zellen zerlegt wurde, gleichgiltig 
welche die weisse oder schwarze war. Ja er fand sogar, dass Eier, die sich mit dauernd nach oben gerichtetem weissen Pol entwickelt hatten, eine weisse Medullarrinne bildeten, dass also infolge der richtenden Schwerkraftswirkung weisses statt schwarzes Material zur Bildung des Centralnervensystems verwendet worden war.

Diese vor nunmehr fast zwanzig Jahren erhobenen Befunde hat bis jetzt niemand systematisch nachgeprüft. B o r n hat seine Analyse nur auf das Verhalten der anormal fixierten Eier bis zum Auftreten der ersten Furche ausgedehut. Er fand, dass in derartig behandelten Eiern eine Umlagerung des Dottermaterials eintrat, die die ursprüngliche Struktur annähernd wieder herstellte. Damit war nun zwar allen weiteren Hypothesen, die Pflüg e $r$ an seine Befunde geknüpft hatte, der Bodell entzogen worden, aber die von Pflüger festgestellte Thatsache der völligen Umkehr des Furchungsmodus - weisse Zellen klein, braune gross - blieb nichtsdestoweniger bestehen und harrte ihrer Erklärung.

O. Schultze berichtet (45) zwar auch, dass er in Compression befindliche Froscheier im Stadium der ersten Furchen (die infolge der Compression alle Meridionalfurchen waren) gedreht, und dass dann diese Eier nach 10 Stunden nach dem aequalen Typus gefurcht gewesen wären. Er hat indessen keine Schnittserien von den so veränderten Eiern angelegt und kann daher über die inneren Vorgänge in diesen Eiern nichts aussagen. Darauf aber kommt es allein an, wenn wir entscheiden wollen, ob eine Induction durch Schwerkraft, oder ein einfacher hydrostatischer Vorgang vorliegt. Aber man kann ihm doch eine gewisse Berechtigung nicht absprechen, wenn er $(45,47,49)$, solange diese von P f 1 ü ge $\mathrm{r}$ gemachten Befunde nicht erklüt sind, den Standpunkt vertritt, dass die Schwerkraft auf die Furchung des Froscheies in ganz bestimmter Weise richtend einwirke. Freilich darf er diese Einwirkung nicht Geotropismus nennen (vergl. 24, pag. 31). Tropismus nennt man in der Reizplysiologie das Wachstum eines Organismus zu einem Reiz hin oder von einem Reiz fort. Das Froschei wăchst aber überhaupt nicht während der Furchung. Wenn von irgend einem Reiz die Rede ist, so kann es sich nul um eine Barymorphose, d. h. „durch die Schwerkratt ausgelöste, formative Wirkungen" handeln (24, pag. 32), die darin bestehen 
würde, dass die Teilungsenergie der jeweils oberen Zellen erhöht, die der jeweils unteren erniedrigt werden würde.

Liegt nun eine solche Barymorphose in der T'hat bei den von Pflüger gemachten Befunden vor?

\section{A. Der makroskopische Befund.}

lch hatte schon in meiner ersten Arbeit (37) die Vermutung ausgesprochen, dass auf dem Vierzellenstadium gedrehte Eiel' deshalb Tagelang unveränderlich in dieser Lage blieben, weil durch die neu auftretenden Zellwande der Druck und del Reibungswiderstand zu gross würden, als dass eine Umlagerung der Dottersubstanzen vor Auftreten der dritten Furche stattfinden könnte. Diese Vermutung versuchte ich experimentell folgendermaassen $\mathrm{zu}$ prüfen. Durch die Untersuchungen von O. Hertwig (29) und O. Schultze (46) ist bekannt, dass niedere Temperaturen die Entwicklung des Froscheies in beträchtlicher Weise aufhalten, ohne dass die Eier irgendwelchen bemerkenswerten Schaden nelimen. Ich legte also auf dem Vierzellenstadium gedrehte, in Plattenzwangslage befindliche Eier in Wasser von 1-2 Grad Warme. Ich hofite, dass hier durch die Külte das Auftreten weiterer I'urchen so lange aufgehalten werden würde, dass der Inhalt der vier Zellen Zeit haben würde, sich seinem spezifischen Gewicht nach zu orientieren. Diese Frwartung ist denn auch in allen Fallen bestatigt worden. Sowohl diejenigen Eier, die sich in Plattencompression befanden und auf dem Vierzellenstadium gedreht worden waren, als auch diejenigen Eier, die in Pflüger'scher \%wangslage auf dem Zweizellenstadium gedreht und dem Einfluss niederer Temperaturen ausgesetzt worden waren, zeigten nach etwa zwanzigstïndigem Verweilen im Eisschrank vollständig den von I' fl ü g e 1 geschilderten Furchungsmodus: Die weissen Hemisphären waren in viele kleine, die schwarzen in wenige grosse Zellen zerlegt worden, wie es Figur 1 und 1a, Tafel XIX zeigt. Ich latte also eine direkte Bestatigung der $\mathrm{Pfl}$ üg er 'schen Befunde vor mir, und zwar ist es, so weit mir bekannt, das erste Mal, dass nach P fl ïg er derartige Befunde in solch prägnanter Weise erhoben worden sind. Ich modifizierte den Versuch nun so, dass ich einen Teil der Eier, die auf dem Vierzellenstadium gedreht worden waren, bei $1-2$ Grad, einen anderen bei 10 Grad und 
eine dritten bei 20 Grad züchtete. Die Resultate waren folgende: Die in der Kälte gezüchteten Eier hatten sich nach etwa zwanzig Stunden nach dem schon oben beschriebenen Furchungsmodus, oben kleine helle, unten grosse dunkle Zellen, geteilt. Die bei 20 Grad gezüchteten Eier, ebenso wie die bei 10 Grad gezüchteten, unterschieden sich nach 11 Stunden in nichts von den bei gleicher Temperatur gezüchteten Kontrolleiern (Fig. 2 und 2a, Tafel XIX). Die dunkle Hemisphäre weist bei beiden kleine, dunkle, die helle grosse, helle 7ellen auf. Die Furchen auf der hellen Hemisphäre sind fast nur Meridionalfurchen, die vom dunklen Pol aus durchgeschnitten haben, latitudinale Furchen sind auf der weissen Hemisphäre nur ganz wenige vorhanden (auf unserer Abbildung sogar nur eine). In klassischer Weise den umgekehrten Furchungsmodus zeigt ein auf dem $/$ weizellenstadium gedrehtes, in Plattencompression bei 10 Grad gezüchtetes Ei (Fig. 3 und 3a). Die hellen Zellen sind klein, die dunkelen gross. Am dunkelen Pol überwiegen die von der hellen Hemisphäre aus durchgeschnittenen Meridionalfurchen, Latitudinalturchen sind in der Umgebung des dunkelen Poles nur spärlich rorhanden.

Verschiedene Eier, die in P f l üger'scher 'Zwangslage auf dem $Z$ weizellenstadium gedreht und bei 1-2 Grad gezüchtet worden waren, lieferten Embryonen mit hellem Medullarrohr, wie sie entsprechend auch von Pflüger beschtieben worden sind (Fig. 4).

Wenn man nun in umgekehrter $Z$ wangslage befindliche Eier, die nach dem normalen Furchungsschema gefurcht sind - braune Zellen klein, helle gross - durch Zufubr von Wasser, respektive Aufheben der Compression, vom Drucke befreit, so sieht man, dass sie nach einiger Zeit wieder in die für das Froschei typische Stellung - braune Hemisphäre nach oben, helle nach 'unten - zurückrotieren. Setzt man aber Eier, die nach dem umgekehrten Furchungsschema gefurcht sind, in Freiheit, so tritt nichts dergleichen ein. Sie bleiben unverrückt in ihrer alten Lage. Bilden solche Eier einen Urmund, so ereignet es sich oft, dass ihr Dotterpfropf ganz oder doch zum Teil braul ist. Es hat also bei letzterer Kategorie von Eiern eine Umlagerung des Dottermateriales stattgefunden, die allerdings von aussen nicht kenntlich ist, bei ersterer Kategorie nicht. 


\section{B. Der mikroskopische Befond.}

Fertigt man von normalen, etwa 11 Stunden nach der Befruchtung fixierten Morulis vom weissen zum schwarzen Pol gehende Schnitte an, so erhält man folgendes Bild, das als typisch zu betrachten ist (Fig. 5). Die um den braunen Pol herum gelegenen Zellen, sowie die seitlichen sind in gauzer Ausdehnung von braunem Pigment erfüllt. Am hellen Pol sieht man auf dem Schnitt zwei grosse, helle Zellen, die im Durchschnitt etwa 2-3 mal so gross sind als die kleinsten Zellen des dunklen Poles. Eine Furchungshöhle ist vorhanden. Die schwarzbraune Pigmentrinde ist am dunklen Pol am stärksten, wird seitlich immer schmaler, um am weissen Pol ganz zu verschwinden, wie das schon von Born (2) gezeigt worden ist. Untersucht man nun Durchschnitte von Eiern, die vor Auftreten der erste $n$ Furche in schiefer Zwangslage fixiert worden sind, so sieht man nach B orn, wie der herabtliessende weisse Dotter die Pigmentrinde zusammengeschoben und auf grössere Strecken von dem Ei abgelöst hat. Die Folge davon ist, dass 'Teile der weissen, peripheren Platte, die beim Absinken des weissen Dotters infolge ihrer zäheren Consistenz stehen geblieben ist, sichtbar werden, die weisse Hemisphäre also scheinbar vergrössert erscheint (vgl. 2). Indem der aufsteigende braune Dotter diese periphere, weisse Platte unterlagert, entsteht der charakteristische, graue Fleck. Betrachten wir jetzt einen schnitt durch ein $\mathrm{Ei}$, das auf dem Zweizellenstadium in Pfliiger'scher Zwangslage gedreht und 20 Stunden lang bei 2 Grad gezüchtet worden ist. Das betreffende Ei (Fig. 6) entspricht etwa dem Stadium der Figur 3. Die am oberen Pol gelegenen Zellen sind klein. Wührend aber bei normalen Morulis die Zellen an den Polen nur eine Art Dotter enthalten, weisen die Zellen hier am oberen Pol beide Dotterarten auf. Nach der Peripherie hin sieht man in jeder der fünf Zellen ein schmales Band von weissem, grobkörnigem Nahrungsdotter. Der Rest ist von feinkörnigem braunen Bildungsdotter erfüllt. An einer Stelle in jeder Zelle ist es zu grösseren Anhäufungen braunen Dotters gekommen, so dass eigenartige Zeichnungen in Form von Sternen, Bändern, Haken zu Stande gekommen sind. Durch die Mitte des ganzen Eies zieht sich ein Streifen braunen Dotters von Pol zu Pol. Dieser Streifen besteht aus fünf kleinen Zellen, von denen die unterste 
am unteren Pol liegt. Daneben liegt eine grosse, weisse Zelle. An die oberen kleinen Zellen stossen jederseits vier grosse, weisse Zellen, die somit die Flanken des Eies einnehmen. Die Pigmentrinde ist am unteren Pol am dicksten, nimmt nach beiden Seiten allmählich ab, um am oberen Pol fast ganz zu verschwinden.

Die Deutung des Präparates ist nicht schwer. In dem auf dem Zweizellenstadium gedrehten und bei 2 Grad gezüchteten $\mathrm{Ei}$ ist der weisse Dotter in jeder Zelle längs der auusseren Wand abgesunken, der braune längs der Scheidewand der beiden Zellen aufgestiegen. Die Bahn des braunen Dotters ist noch deutlich zu erkennen. Am oberen Pol hat er sich unter der beim Absinken des weissen Dotters stehen gebliebenen peripheren Platte ausgebreitet. Infolge des grossen Widerstandes der dem Absinken des weissen Dotter's in dem in Pfl üger'scher Zwangslage befindlichen $\mathrm{Ei}$ entgegensteht, geht dieses Absinken sehr langsam vor sich; es gelingt ja überhaupt nur dann dieses Phänomen bei $\mathrm{Pfl}$ lüg e reiern nach der ersten Furche hervorzurufen, wenn man durch Kaltewirkung den Ablauf der Furchung erheblich verzögert. Sei es nun, dass hierdurch die Wucht des abströmenden Dotters eine geringere wird, sei es, dass die Pigmentrinde Zeit hat auszuweichen, auf jeden Fall findet das bei Zwangslageneiern vor der ersten Furche von Born konstatierte Zusammenschieben der Pigmentrinde nicht statt, dieselbe bleibt vielmehr an ihrem Platze. So sind die jetzt unten befindlichen, weissen Zellen von einer dicken, braunen Pigmentrinde bedeckt, während die oberen, bis auf die weisse periphere Platte, ganz. aus braunem Bildungsdotter bestehenden Zellen keine Pigmentrinde besitzen. Es wird also bei makroskopischer Betrachtung der Anschein hervorgerufen, als ob oben weisse Zellen lagen und unten braune. In Wahrheit liegen aber, wie beim normalen Verhalten, oben braune Zellen, die nur durch die beschriebene, weisse Schicht verdeckt sind, und unten weisse, die aber durch die dicke, braune Pigmentrind verborgen werden. Die Umkehr des Furchungsmodus, wie ihn Pflüger beschreibt und auf die sich 0 . Schultze $(45,47,59)$ verschiedentlich ausdrücklich beruft, allerdings nicht auf Grund systematischer, durch Untersuchungen von Schnittserien gestützter Experimente, ist also nur eine scheinbare. Auch hier, wie bei normaler Furchung, befindet 
sich im Grossen und Ganzen da, wo die kleinsten 7ellen sind der braune Bildungsdotter und da, wo die grossen Zellen sind der weisse Nahrungsdotter.

Auch die Erscheinung der weissen Medullarwülste ist nicht etwa die Folge davon, dass durch den richtenden Einfluss der Schwerkraft in diesem Falle ausnahmsweise die Medullarwülste sich aus dem Material der weissen Hemisphüre gebildet haben, statt wie gewöhnlich aus dem der schwarzen. Fig. 7 zeigt einen Querschnitt durch einen Embryo, dessen einer Medullarwulst braun, dessen anderer Medullarwulst aber weiss oder vielmehr grau war. Der Embryo stammte von einem Ei, das in Pflüg er'scher Zwangslage auf dem Zweizellenstadium gedreht und bei 2 Grad gezüchtet worden war. Aus diesem Ei entstand keine Doppelbildung, sondern ein fast normaler Embryo. Auf die Analyse dieses Falles will ich hier nicht eingehen. Der Schnitt zeigt sehr schön, dass der linke Medullarwulst von einer dicken, braunen Pigmentrinde bedeckt ist, während sie auf der rechten Seite vollständig fehlt. Dagegen ist in der Grösse der Zellen rechts und links kein Unterschied wahrzunehmen, ebensowenig wie in ihrem Dottergehalt.

\section{Theoretische Schlussfolgerungen.}

Die eben mitgeteilten Befunde bestätigen also die von Pflüger 1883 veröffentlichten Thats achen. Freilich werden wir sie anders deuten müssen, als es Pflüger seiner Zeit gethan hat. Bor'n hat sein Hauptangenmerk auf die Richtung der ersten Furchen, respektive auf die Lage der Kernspindeln gerichtet (l. c. 1 und 2). Er zeigte, dass die stete Coincidenz der ersten beiden Furchen mit der Richtung der Schwerkraft nicht die Folge eines von dieser ausgeiibten Reizes, sondern die Folge einer Umlagerung des Dottermaterials auf rein physikalischer. Grundlage ist. Mit der von Pfl üger mitgeteilten Thatsache der Umkehr des Furchungsmodus hat er sich nicht weiter beschaftigt. Und doch hat P f l ü g e r grad auf diesen Punkt grossen Wert gelegt, wie aus einer Bemerkung in seiner dritten Schrift (42, pag. 9), die nach Borns vorliufiger Mitteilung (1) erschien, und die sich offenbar auf Born bezieht, hervorgeht. Auch O. Schultze hat diese Beobachtung Pflügers öfter zur Stütze der von ihm vertretenen Ansicht, dass die Schwerkraft richtend 
und regulierend auf die Zellteilung einwirke, herangezogen, ohne freilich, wie bereits gesagt, dieselben einer kritischen Nachprüfung unterzogen zu haben. Freilich das makroskopische Bild ist sehr bestechend, so bestechend, dass P fl ü ge r sich angesichts dieses Phänomens zu dem Ausspruch veranlasst sah : „Niemand kann die Ursache dieser merkwürdigen Erscheinung sagen. Niemand hat hier die Berechtigung von primären und sekundären Wirkungen der Schwerkraft zu sprechen" (42, pag. 9). Das iiberaus' klare mikroskopische Bild giebt uns denn doch wohl die Berechtigung, bier von durchaus sekundären Wirkungen der Schwerkraft zu sprechen.

\section{Ka pitel.}

\section{Einfiuss der Schwerkraft auf die Organbildung.}

In dem kiu'z nach der Befruchtung sich in physiologischer Zwangslage befindlichem J'roschei findet, wie wir oben gesehen haben, durch Schwerkraftswirkung eine Ddtterumlagerung statt, durch welche das Eimaterial symmetrisch zu nur einer Symmetrieebene orientiert und so die Medianebene des künftigen Embryos bestimmt wird. Aber nicht nur die Bilaterialität des Embryos wird durch diesen Vorgang festgelegt, sondern auch seine genaue Lokalisation und seine Poluritat. Der Embryo liegt stets auf der Seite, auf der der weisse Dotter abgesunken ist, niemals auf der gegenüberliegenden, auf der der braune Dotter aufgestiegen ist (Roux [44], Born [1, 2], O. Schultze [45, 47, 48, 49], Hertwig [26, 27], Morgan [32] u. a.). Der Kopf des Embryos liegt etwa 15 Grad nördlich des Aequators, der Schwanz 105 Grad südlich davon (Wilson [52], Moszkowski [38 $]^{1}$ ). Es wird also, worauf ich noch einmal ausdrücklich hinweisen will, durch einen äusseren Faktor, Schwerkraftswirkung, die Bilateralität, Polaritat und Lokalisation des Embryos festgelegt. Der virtuelle Embryo ist am befruchteten, aber ungefurchten Froschei vollständig zu bestimmen, wovon ich mich auch durch zahlreiche

${ }^{3}$ Diese Arbeit war schon abgeschlossen, als mir die Arbeiten von H elen Dean King: Experimental Studies of the Formation of the Embryo of Bufo lentiginosus Arch. f. E. M. Bd. XIII, 4, und von Albert C. Eyloshymer: The Formation of the Embryo of Necturus, with Remarks on the Theory of Concrescence, Anat. Anz. 21, in die Hände kamen. Diese beiden Autoren machen für Bufo und Necturnus ungefähr dieselben Angaben, wie Wilson für Chlorophilus und ich für Rana fusca. 
Anstichversuche überzeugt habe. Sticht man mit der heissen Nadel das Ei kurz vor oder nach den ersten Furchen direkt am scbwarzen Pol an, zerstört also das dort gelegene Material, so liegt der Defekt, wenn er nicht überhaupt verheilt, was ziemlich häutig vorkommt, an der Bauchseite des Embryos. Sticht man an der oberen Grenze des grauen Feldes direkt in der Mitte an, so findet sehr häufig eine Differenzierung im Organe überhaupt nicht statt. und man erhält Bilder, die völlig der Figur 6 von Kopsch (30, p. 118) gleichen (Fig. 8). Die Urmundränder haben sich längs einer sagittalen Linie zusammengelegt. Die drei Keimblätter sind vorhanden, von einer Differenzierung in Medullarplatte, Urwirbel Chorda etc. ist keine Spur zu erblicken. Sticht man etwas weiter seitlich an der oberen Grenze des grauen Feldes an, so erhält man Halbbildungen, die jedoch eine ganze Gehirnplatte besitzen (Fig. 9). Sticht man noch weiter seitlich an, so sind die Organe auf der operierten Seite zwar vollständig vorhanden, aber schwächer entwickelt wie anf der anderen Seite. Ein interessantes Beispiel dafür liefern Fig. 10, $10 \mathrm{a}, 10 \mathrm{~b}$ und $10 \mathrm{c}$. Figur 10 stellt einen Embryo dar, der im Achtzellenstadium seitlich von der oberen Grenze des grauen Feldes angestochen worden war. Man sieht, besonders auch auf dem Querschnittbild $10 \mathrm{a}$, dass der linke Medullarwulst bis auf die hintersten Partien ebenso gut entwickelt ist als der rechte. Nach hinten zu wird er jedoch bedeutend schwächer, was namentlich auf dem Querschnitt (10 b) sehr gut zu sehen ist. Am caudalen Ende des Embryos befindet sich auf der linken Seite ein Defekt, der fast wie ein Urmund aussieht; wie der Querschnitt (Fig. 10 c) lehrt, betrifft dieser Defekt nicht mehr den linken Medullarwulst, sondern liegt ausserhalb desselben. Wir werden diesen Befund sofort im Zusammenhang mit den übrigen analysieren, fahren vor der Hand aber in der Beschreibung des rein Thatsächlichen fort. Sticht man an der unteren Grenze des grauen Feldes an, so erbält man ausnahmslos Embryonen mit Spina bifida, bei denen sowohl die Gehirnplatte als auch die Aftergegend wohl entwickelt sind (Fig. 11). Der Defekt kann übrigens nachträglicn überwachsen werden, wie das auch von Hertwig beschrieben worden ist (Urmund und Spina bifida). Sticht man endlich an der dem grauen Felde gegenüberliegenden Grenze von weisser und schwar\%er Hemisphäre an, so erhalt man 
einen Defekt in der Aftergegend des Embryos, der gleichfalls nachträglich überwachsen werden kann (Fig. 12). Diese Befunde zeigen also, dass der Embryo am befruchteten aber ungefurchten resp. in den ersten Furchungsstadien befindlichen Ei bereits völlig lokalisiert ist, und bilden zugleich eine völlige Bestätigung der von Kopsch vertretenen Ansicht über die Bildung der Embryonalanlage bei den anamnioten Wirbeltieren (30, pag. 125, 31, pag. 76). Die untere Grenze des grauen Feldes entspricht der Stelle, wo die erste Anlage des Urmundes stattfinden wird. Sticht man hier an, so entsteht eine Spina hifida oder Anentoblastia ( $\mathrm{R} \circ \mathrm{ux}$ ), weil an dieser Stelle das abgetötete Material ein Hindernis für die Einstïlpung der dorsalen Urmundlippe bildet. Die Herausdifferenzierung des Embryos wird aber hierdurch nicht betroffen. Dieselbe geht von einem Bezirk nördlich von der ersten Urmundanlge ans, die der oberen Grenze des grauen Feldes entspricht. Aus der Mitte dieses Bezirkes entsteht der Kopf, während die seitlichen Partien dem Wachstumszentrum von Rumpf und Schwanz entsprechen. Es findet daher bei Zerstörung der mittleren Partie des grauen Feldes überhaupt keine Differenzierung statt, bei Zerstörung der seitlichen Partien eine Halbbildung mit ganzem Kopf. Der gesamte Rest des Eies beteiligt sich nur insofern an der Bildung des. Embryos, als 7ellen, die nicht zum Embryobildenden Bezirk gehören, bei der Zusammenziehung der seitlichen Urmundränder in denselben gelangen und dann zum Aufbau des Embryos verwandt werden. Daher kommt es, dass, wie das schon Kop sch durch Anstichversuche $\mathrm{nach}$ Auftreten des Urmunds festgestellt hat, die Medullarwïlste anf der operierten Seite zwar gebildet werden aber schwächer ausfallen als auf der intakten Seite. Der Defekt neben dem caudalen Ende des Medullarrohres, wie ihn Figur 10 und $10 \mathrm{c}$ zeigen, ist dadurch entstanden, dass durch das abgetötete Material die Bewegung der linken Urmundlippe hier in ahnlicher Weise aufgehalten worden ist, wie wir das bei Fig. 11 für die Bewegung der dorsalen Urmundlippe konstatierten ${ }^{1}$ ). Da

1) Zur Methodik der Anstichversuche mache ich noch einmal ausdrücklich darauf aufmerksam, dass man unbedingt anf die Lage der angestochenen Eier achten muss, weil durch das auftretende Fxtraovat die Eier oft in schiefer Zwangslage fixiert werden, wodurch natürlich das Resultat erheblich modifiziert wird, am besten ist es erst auf dem Vierzellenstadium 
das graue Feld, wie als bewiesen anzusehen ist, durch Schwerkraftswirkung entsteht, so ist an einer Lokalisation morphogenetischer Vorgänge durch Schwerkraftswirkung wohl nicht mehr zu zweifeln. Es fragt sich nun, wie diese Wirkung zu Stande kommt. Sehen wir uns zunächst nach analogen Fällen in der Ontogenese anderer Tiere und Pflanzen um, so kommen allein die Beobachtungen Leitgebs (32) in Betracht, auf die auch schon Pflüger verwiesen hat. Leitgeb hat gefunden, dass die Richtung der ersten Teilung der keimenden Spore von Marsilia, durch welche die stammbildenden T'eile voll den Wurzel bildenden geschieden werden von der Schwerkraft bestimmt wird. Allein Herbst (23, pag. 736) bemerkt ganz richtig, dass es sich hier um eine reine Induktion durch Schwerkraft nicht handeln könne, da, wie Leitgeb selbst angiebt, die erste T'eilung auf jeden Fall durch die Archegonachse hindurchgehen muss, in einer Richtung also schon a priori fixiert ist.

Von anderen äusseren Reizen, die, im Beginn der Ontogenese wirkend, bestimmt lokalisierenden Einfluss ausüben, ist die von Stahl aufgefundene Wirkung des Lichtes auf die Teilung von Equisetumsyoren zu nennen (50). Stahl fand, dass die Kernspindeln einseitig belichteter Equisetumsporen sich parallel zur Richtung der Lichtstrahlen stellten. Von den durch die 'Teiluug hervorgehenden beiden Zellen wird die dem Licht abgewandte zur Rhizoidenzelle. Winkler fand, dass die Eier von Cystosira barbata, einer Alge, in gleicher Weise auf die Lichtwirkung reagierten (53), und berichtet, dass Rosenvinge, sowie Farmer und Williams das gleiche Verhalten bei einigen Fucaceeneiern fanden. Bei tierischen Eiern ist eine ähnliche Wirkung des Lichtes, soweit daraufhin untersucht worden ist, bis jetzt nicht nachgewiesen worden. Da nun die Eier der oben

anzustechen, weil dann die Struktur des Eies soweit fixiert ist, dass ein Absinken des weissen Datters nicht mehr za befürchten ist.

Den Unterschied zwischen den Anstichversuchen von $R \circ u x$ und den meinen besteht darin, dass Roux die Kerne tötete und so bewirkte, dass die Hälfte, resp. ein Viertel des Eies sich überhaupt nicht entwickelten; während ich unter möglichster Schonung der Kerne nur verhältnismässig geringe Mengen Protoplasma abtötete. Da nun diese toten Massen in loco blieben, so verhinderten sie, dass die normaler Weise an diesen Stellen vor sich gehenden Differenzierungen erfolgen konnten. 
genannten Pflanzen zahlreiche Chromatophoren besitzen, so glaube ich mit Herbst (23, pag. 732), dass in ihrem Verhalten bei der ersten Teilung keine Photomorphose zu erblicken ist - d.h. keine morphologische Reizwirkung des Lichtes - sondern, dass es sich um eine heliotactische Erscheinung handelt. Analog, wie durch Schwerkraftswirkung beim Froschei eine Scheidung der verschieden spezifisch schweren Substanzen erfolgt, so werden durch Lichtwirkung die verschieden Licht empfindlichen Substanzen der betreffenden Pflanzeneier in chlorophyllhalt ge, positiv heliotactische und chlorophyllarme, negativ heliotactische, geschieden.

In letzter Linie sind noch die ausserordentlich wichtigen Veränderungen in der Entwicklung der Seeigeleier zu berücksichtigen, die C. Herbst durch Zusatz geringer Dosen von $\mathrm{Li} \mathrm{Cl}$ zum Meerwasser hervorgerufen hat $(19,20,21)$. Normaler Weise geht die Urdarmbildung der Seeigeleier von einem nur kleinen Bezirk der Blastula aus. Bei Anwesenheit von $\mathrm{Li} \mathrm{Cl} \mathrm{im}$ Meerwasser vergrössert sich dieser Bezirk proportional dem Prozentgehalt an Lithium, den das Wasser enthäl, so dass in dem extremsten Fallen die gesamte Blastula Urdarm bildend wird, das Ektoderm also völlig unterdrückt wird. In diesen Fällen kommt es natürlich zu keiner Einstülpung.

Aber auch in den in schwächeren Lithiumlösungen gezüchteten Eiern findet keine Finstülpung statt, sondern es kommt zu einem Wachstum in entgegengesetzter Richtung, zur Bildung der von Herbst recht treffend so genannten Exogastrula. Herbst und Driesch erklären diese Frscheinung so, dass - kurz zusammengefasst - nicht etwa das Lithium einen direkt formverăndernden Reiz auf den Echinidenkeim auszu üben vermag, sondern dass durch die Lithiumwirkung primär die Struktur des Eies geăndertwird und dass der so veränderte Keim auf den sonst die Bildung des Entoderms auslösenden innern Reiz in anderer Weise reagiert als normal.

Diese Erklärung scheint mir auch auf die anderen oben beschriebenen Falle durchaus zuzutreffen. Durch einseitige Belichtung wird z. B., wie wir sahen, erst die Struktur der Equisetumsporen modifiziert, die so verănderte Struktur bedingt aus innern Gründen eine bestimmte Stellung der Kernspindeln u. s. f. Wir werden sehen, dass sich auch die Wirkung 
der Schwerkraft auf die Entstehung der bilateralen Symmetrie des Froscheies nur unter diesem Gesichtspunkte begreifen lässt. Dennoch besteht ein Unterschied zwischen der Wirkung des Lithiums einerseits und der Wirkung des Lichtes auf die Equisetumsporen andererseits. Die Anwesenheit von Lithium nämlich im Seewasser ist zur Erzielung von Lithiumlarven absolut notwendig; nicht aber die einseitige Belichtung für die Entwicklung der Equisetumsporen. Dieselben entwickeln sich auch bei allseitiger Belichtung oder im Dunkeln, allerdings etwas langsamer. Diese Reize fallen, nach der Einteilung von C. Herbst, die ich als eine ausserordentlich glückliche ansehe, unter die Kategorie I, bloss lokalisierende, nicht notwendige. Jene dagegen fallen unter die Kategorie $\mathrm{II}_{\gamma}$, strukturelle Reize, welche sich dadurch charakterisieren, dass sie „das Ürsachengetriebe in seinem Zusammenhang afficieren" (23, pag. 822). Um welche von diesen beiden Reizkategorien handelt es sich nun in unserem Falle? Die beiden anderen Herbst'schen

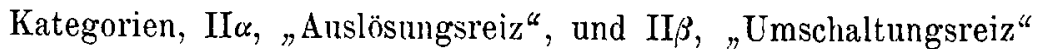
können wir als selbstverstandlich nicht in Betracht kommend übergehen.

Nur ad vocem Geotropismus möchte ich noch einige Worte sagen, weil O. Schultze (47) "die Sorge“ des Froscheies sich durch die Perivitellinausscheidung seine Stellung zur Gravitation zu sichern als eine Fühigkeit ansieht, die man seiner Meinung nach als Geotropismus bezeichnen könnte. Unter Geotropismus versteht man einen komplizierten Reizvorgang. dessen Geschehen an die Anwesenheit gewisser speziell differenzierter Organe gebunden ist. Das noch undifferenzierte Ei besitzt aber naturgemass derartige Organe noch nicht, kann infolge dessen also auch auf äussere Reize nicht in spezitischer Weise. reagieren. Die einzige Form, in welcher lebendes Protoplasma auf der niedersten Differenzierungsstufe auf aussere Reize zu reagieren vermag, mögen es nun elektrische oder chemische, oder sunst welche Reize sein, ist durch Ausdehnung oder Zusammenziehung. In dieser Beziehung, wohlgemerkt nur in dieser, steht das tierische oder pflanzliche Ei auf keiner höheren Stufe als die niederste Amöbe. Die Struktur eines Vertebraten Eies ist natürlich mendlich komplizierter als die einer Amöbe - enthät es doch potentia den gesamten zukünftigen 
Organismus in sich - Aber eine Differenzierung in Organe und damit die Möglichkeit in spezifischer Weise auf äussere Reize zu reagieren, können wir dem Ei ebensowenig zuschreiben, wie der Amöbe. Während der Furchung aber findet keine Differenzierung statt. Der abgefurchte Keim ist darum als System genau dasselbe wie das befruchtete Ei. Hier einige Beispiele, um diese Behauptung zu erhärten: Bringt man Froscheier $\mathrm{nach}$ der dritte $\mathrm{n}$ Furche, welche den Bewegungen des Eidotters innerlualb des Eies ein definitives Ziel setzt, in irgend eine beliebige Lage zur Richtung der Schwerkraft und fixiert sie in derselben, so geht die Entwicklung ungestört weiter, ohne dass irgend welche Veranderungen auftreten. Derartige Eier bilden auch noch den Urmund in normaler Weise und entwickeln sich, wenn man sie jetzt, d. h. nach Bildung der ventralen Urmundlippe, in Freiheit setzt, zu ganz normalen Embryonen. Lässt man die Zwangslage aber auch jetzt noch bestehen, so sterben sie ab, falls die Kopfanlage nach abwärts orientiert ist, entwickeln sich aber zu völlig normalen Larven. wenn das nicht der Fall ist. Grade auf letzteren Punkt lege ich ganz besonderes Gewicht namentlich gegenüber O. Schult ze. Ich habe wenigstens 50 Embryonen, deren totale Zwangslage ich genau nach den von O. Schultze angegebenen Cantelen geprüft habe, und die sich trotzdem ganz normal entwickelt haben. Nur, wie gesagt, wenn die Kopfanlage nach abwärts orientiert ist, sterben die Eier auf dem Stadium des kreisförmigen Urmunds $a b$, also zu einer Zeit, wo die ersten Differenzierungen schon vor sich gegangen sind und sich auch speziell die nervösen Zentralorgane schon in situ befinden. Auch das Verhalten der Eier auf dem Zentrifugalapparat gehört hierher. Die Furchung stockt auf dem Zentrifugalapparat selbst bei einer Beschleunigung nicht, die $10 \mathrm{mal}$ so gross ist als die Beschleunigung durch Schwerkraftswirkung. Das Ungeteiltbleiben des weissen Dotters ist natürlich auch keine Folge irgend einer Reizwirkung, sondern auch nur eines rein mechanischen Vorgangs. Die Dotterelemente werden eben aus dem Protoplasma heraus an die Peripherie geschleudert, grade wie beim Zentrifugieren von Milch die Fettkügelchen aus der Flüssigkeit sedimentiert werden. Ueber die ersten Anfänge der Gastrulation vermögen aber die Eier nicht herauszukonimen, so lange sie 
sich in Rotation befinden. Auch die Herbst'schen Lithiumeier weisen bis zum Blastulastadium keinerlei Störungen auf. Erst mit beginnender Gastrulation machen sich die formverändernden Wirkungen des Lithiums geltend. Ich glaube, diese Beispiele, die sich leicht noch vermehren liessen, genügen, um unsere Behauptung zu erhärten, dass wahrend der Furchung äussere Reize irgend welche spezifische Wirkungen nicht ausüben können, da zur spezifischen Reaktion speziell differenzierte Organe gehören, die dem sich furchenden $\mathrm{Ei}$ noch mangeln. Dass aber in Sonderheit zur Perception des Schwerkraftreizes nicht nur bei Tieren, sondern auch bei Ptlanzen ganz bestimmt gebaute Organe nötig sind, geht aus den neuesten Arbeiten auf botanischem Gebiet hervor. Nĕmec (39) und Haberlandt (17) haben unabhängig von einander die Behauptung aufgestellt, dass die Perception des Schwerkraftreizes durch analog den Otoresp. Statocysten gebaute Organe vermittelt würde. Als diese Organe sind von beiden übereinstimmend die Stärkescheiden bezeichnet worden, wobei den Stärkekörnern die Rolle der Oto- resp. Statolithen zugeschrieben wurde. Diese Behanptung ist nun durch $\mathrm{Haberlands}$ letzte Publikation (18) in meiner Meinung nach einwandfreier Weise, durch das Experiment bestătigt worden. Es gelang ihm nämlich zu zeigen, dass Teile von Pflanzen, die sonst kräftig geotropisch reagierten (linum perenne), im Winter, wenn ibre Stärke völlig verschwunden war, den Schwerkraftsreiz nicht mehr zu perzipieren vermochten, diese Fähigkeit aber sofort wieder erlangten, wenn sie nach mehrstündigem Aufenthalt im warmen Zimmer die. Stärke neu gebildet hatten. Auf Grund dieser Ueberlegungen wird es uns auch sofort klar, warum die ausseren Reize, die wahrend der späteren Entwicklung der Organismen, speziell der Pflanzen, eine so grosse Rolle spielen, während der ersten Entwicklungsstadien ganz in den Hintergrund treten.

Wir können also nicht sagen die Schwerkraft induziert die Bilateralităt des Embryo. Die Schwerkraft kann direkt nur rein physikalisch auf das Ei wirken, durch Umlagerung seiner Dottersubstanzen. Durch diese Einwirkung auf das eben befruchtete Ei wird die Struktur desselben verändert. War es vorher radiär gebaut und isotrop, so erhält es jetzt einen anisotropen, bilateralen Bau. Dieser bilaterale Bau wird wăhrend 
der Furchung nicht nur festgehalten, sondern auch fixiert. Hierin scheint mir überhaupt neben der Zerkleinerung des Eidotters und Vermehrung der Kerne der Wert der Furchung zu bestehen. Die Struktur des befruchteten, ungefurchten Eies ist eine labile. Dieser labile Zustand wird durch die Furchung in einen stabilen übergeführt. Dies ist der wesentliche Unterschied zwischen dem befruchteten, ungefurchten $\mathrm{Ei}$ und dem abgefurchten Keim. Daher müssen Struktur verändernde Reize vor oder im Beginn der Furchung angreifen. Nachher Iässt sich die Struktur des Eies nicht mehr verändern. Herbst erzielte nur dann Lithiumlarven, wenn er die Seeigeleier kurz nach der Befruchtung oder während der ersten Furchungen in Lithiumwasser brachte, spätere Einwirkung des Lithiums hatte keinen Erfolg. „Die morphologische Wirkung des Lithiums kann sich an befruchteten Seeigeleiern um so weniger geltend machen, je weiter sie in der normalen Entwicklung fortgeschritten sind" (20, pag. 177). Wir haben gesehen, dass ebenso die Schwerkraft nur so lange verändernd auf die Entwicklung des Froscheies einwirken kann, als sie im Stande ist die Struktur des Eies zu verändern, also spätestens bis zum Auftreten der dritten Furche.

Aus unseren Ausführungen geht also hervor, dass wir die Wirknng der Schwerkraft auf die Entwicklung des Froscheies als strukturellen Reiz auffassen müssen. Das unbefruchtete Froschei besitzt eine gewisse Struktur. Diese Struktur wird kurz nach der Befruchtung durch Schwerkraftswirkung geündert. Die so verinderte Struktur ist derartig, dass sie auf den (innern) Organbildung auslösenden Faktor in der typischen Weise reagiert. Das unbefruchtete $\mathrm{Ei}$ ist radiär gebaut und ist völlig isotrop. Jeder Meridian ist jedem anderen gleichwertig. Dieser radiăre Bau wird durch die Wirkung der Schwerkraft bald nach del Befruchtung in einen bilateralen verwandelt. Dadurch wird die Isotropie des Eies zerstört. Das befruchtete Froschei besitzt einen anisotropen Bau. Es ist nun sehr interessant, dass ebenso, wie das unbefruchtete Froschei gegen den strukturverăndernden Einfluss der Schwerkraft bis zu einem gewissen Grade gefeit ist, auch das unbefruchtete Seeigelei auf den strukturverändernden Einfluss des Lithiums nicht zu reagieren vermag. Herbst hat unbefruchtete Seeigeleier bis $31^{1 / 2}$ Stunde 
im Lithium liegen lassen; brachte er sie dann in reines Seewasser und befruchtete sie dort, so entstanden stets völlig normale Plutei aus ihnen. Es ist also wohl die Anisotropie des befruchteten Echinodermenkeimes gleichfalls keine präformierte, sondern erst nach der Befruchtung erworben. Angesichts der formativen Wirkung des Lithiums liegt der Schluss sehr nahe, dass die diese Anisotropie schaffende, aussere Kraft, nicht wie beim Froschei physikalischer, sondern chemischer Natur ist.

Es bleibt uns jetzt noch ïbrig, festzustellen, auf welchem Wege nun die Bilateralität des Keimes die Bilateralität des Embryos zur Folge hat. Vorher aber müssen wir noch einige kurze Worte über den Grundcharakter der Entwicklung sagen. Ich stehe in dieser Beziehung roll und ganz auf dem Boden der von $\mathrm{H}$ ans Driesch in seinen ersten Schriften $(3,6,7,8,11)$ aufgestellten Theorie. Dieselbe lisst sich kur'z folgendermassen zusammenfassen: „Die Summe der ontogenetischen Möglichkeiten, gleichsam das die Summe der Speziescharaktere reprisentierende Magazin: kurz die wahre Anlagesubstanz, wenn man das Wort recht verstehen will, muss als im $\mathrm{K}$ ern der Eizelle liegend angenommen werden" (11, p. 103). Dass also aus einem Froschei ein Frosch, aus einer Eichel ein Eichbaum wird, dafür liegen die Gründe im Kern. Die Torbedingungen aber für-die Realisierung dieser Möglichkeiten liegen in der Organisation des Eies „indem in ihr die Ursachen gegeben sind, welche die sich als erste entfaltenden Möglichkeiten auslösen und damit erst den sich ferner entfaltenden die Vorbedingungen ihrer Realisierung schaffen." (ibid.) I)as heisst mit anderen Worten: Je de l Kern besitzt potentia die Fahigkeit in sich, den gesamten Organismus aus sich hervorgehen zu lassen. Die Organisation des befruchteten Eies aber ist eine anisotrope. Dieser anisotrope Bau des Eiprotoplasmas wirkt als Reiz auslösende Ursache bestimmend auf die Entfaltung der ersten Organe -wir werden gleich sehen in welcher Weise - (primarer Elementarvorgang nach $\mathrm{Driesch}, 8$, pag. 71). Diese primären Organe wiederum lösen die Entwicklung sekundärer Organe aus u. s. f. Es längt also die Lokalisation der Organe sowie der Rythmus ihrer Entwicklung vom Bau des Eies ab. Es liegt in den uns verborgenen Qualitäten des Kernes begründet, 
dass die einzelnen Organe des Frosches ihren spezifischen nur dem Frosche zukommenden Charakter besitzen, in der des Eiprotoplasmas aber liegt der Grund davon, dass aus dieser Zelle eine Sinneszelle und keine Drüsenzelle, aus jener eine Ganglien- und keine Bindegewebszelle geworden ist. „Die prospective Bedeutung der einzelnen Blastomeren ist eine Funktion ihrer relativen Lage." (6, pag. 243).

Nachdem wir uns über diese allgemeinen Gesichtspunkte klar geworden sind, kehren wir wieder zu unserem eigentlichen Problem zurück: auf welche Weise bestimmt die Schwerkraft die Bilateralität, Polarität und Lokalisation des Embryos? Rekapitulieren wir noch einmal, was bis jetzt feststeht: Das unbefruchtete $\mathrm{Ei}$ ist isotrop, durch Schwerkraftswirkung erhalt es kurz nach der Befruchtung die Symmetrieebene und wird auf diese Weise anisotrop. Inwiefern bewirkt nun diese Anisotropie die Entfaltung der primaren Organe - denn um diese kann es sich nach obigen Ausführungen allein handeln - an ganz bestimmt lokalisierter Stelle? Betrachten wir einmal einen schematischen, medianen Sagittalschnitt durch ein Froschei kurz vor Auftreten der dritten Furche.

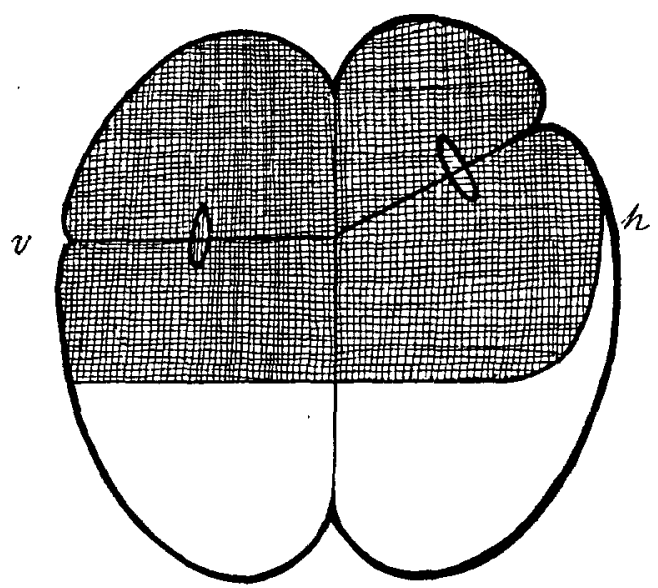

Wir sehen, dass durch die zweite Furche das Ei in zwei ungleiche Hälften geteilt worden ist, indem in den hinteren (b) Zellen der weisse Nahrungsdotter höher hinaufreicht als in den vorderen (v). Wie werden sich in diesem Ei die Kernspindeln zur nächsten Teilung einstellen müssen? In jeder der beiden 
vorderen Zellen müssen sich die Kernspindeln, um in der Richtung der. grössten Protoplasmamenge zu stehen, parallel zur zweiten Furche stellen und näher dem schwarzen Pol. Es resultiert aus dieser Stellung der Kernspindeln eine näher dem schwarzen Pol liegende Latitudinalfurche, welche senkrecht auf erster und zweiter Furche steht. Anders liegen die Verhăltnisse in den hinteren Zellen. Hier ist die Richtung der grössten Protoplasmamenge durch die periphere weisse Platte, die bei Absinken des weissen Dotters stehen geblieben ist, verändert worden. Infolgedessen stellt sich die Kernspindel nicht parallel sondern schrag $\mathrm{zu}$ der zweiten lurche ein, wie das Schema zeigt. Die Zellteilung verläuft also schräg zur zweiten Furche, ein Factum, von dessen Richtigkeit man sich leicht durch den Augenschein überzeugen kann. Diese schrüge Stellung der dritten Eurche, verbunden mit dem Auftreten des grauen Feldes, haben zu der irrtümlichen Ansicht geführt, als ob die Eier von R. fusca und R. esculenta schrïg zur Verticalen orientiert wăren. Die Ei er sind in Wahrheit vollstandig senkrecht gestellt, wie das ja auch a priori selbstverstandlich ist. Man darf eben nicht übersehen, dass das grane Feld zur schwarzen und nicht zur weissen Hemisphäre gehört, und dass die dritte Furche keine Latitudinalfurche, sondern eine Schrägfurche ist. Man kann sich von dieser Thatsache schon dadurch überzéugen, dass die hinteren oberen Zellen immer kleiner sind als die vorderen oberen Zellen, worauf schon $\mathrm{Roux}^{1}$ ) (44, pag. 112/113) aufmerksam gemacht hat, ohne freilich dieser Sache irgend welchen Wert beizulegen. Und doch ist meines Erachtens, der kleinere Umfang der hinteren oberen Zellen eine Thatsache von fundamentaler Wichtigkeit. Wenn wir die Gleichwertigkeit aller Furchungskerne, woran wohl nicht mehr zu zweifeln ist, zugeben, so müssen wir auch annehmen, dass ihre Teilungskraft eine gleiche ist. Bei gleicher Teilungskraft werden aber diejenigen Zellen sich am schnellsten teilen, bei denen der zu überwindende Widerstand, d. h. die zu bewaltigende Protoplasmamenge am geringsten ist. In der That

1) Roux giebt diese Thatsache nur für $R$. esculenta an. Wenn er sagt, dass bei $R$. fusca das Furchungsbild einerseits zu regelmässig, andrerseits zu unregelmässig ist, um ein Schema aufzustellen, so trifft das, wie schon 0 . Schultze (48) gezeigt hat, nicht za. Das Furchungsbild von $R$. fusca ist dem ron $R$. esculenta durchaus identisch. 
sehen wir, dass die Furchen 4. Ordnung unter normalen Ver: băltnissen stets zuerst in den hinteren, oberen Zellen auftreten es sind Meridionalfurchen, - erst fünf bis zehn Minuten später erscheinen sie in den vorderen, oberen Zellen. Dieser Vorsprung wird natürlich bei jeder folgenden Teilung immer grösser, so dass wir im Blastulastadium an dieser Stelle des Eies, die äusserlich der Stelle des grauen Feldes entspricht, die kleinsten Zellen vorfinden, was schon von 0 . Schultze konstatiert worden ist, der indessen irgend eine Erklärung für diesen. Umstand nicht zu geben vermochte. (48)

Ein zweiter Grund dafür, dass an dieser Stelle die Furchung ganz besonders schnell vor sich geht; ist folgender. Wahrend sonst im ganzen Ei weisser und brauner Dotter ohne scharfe Grenze in einander übergehen, stossen an dieser Stelle des Eies reiner Bildungsdotter und reiner Nahrungsdotter unmittelbar an einander. Es kann keinem Zweifel unterliegen, dass dieser letztere wähend der Furchung assimiliert wird. Man kann sich durch direkte Beobachtung davon überzeugen, dass die graue Stelle während der Furchung immer dunkler wird, bis sie im Blastulastadium restlos in die braune Hemisphäre übergeht.

Nun laben Driesch am Seeigel- und Ascidien-Ei $(9,15)$, Herlitzka am Ei von Triton (25) Morgan bei Amphioxus (35) nachgewiesen, dass die Grösse der Zellen einzelner spezifischer Organe eine fest fixierte ist.' Ich stelle mir deshalb die ersten Differenzierungsvorgänge beim Froschei folgendermassen vor. Die in der Umgebung des grauen Feldes gelegenen Zellen gelaugen infolge ibrer rascheren Teilungen früher als alle anderen Zellen an die Grenze ihrer Teilbarkeit, unter die sie infolge ihrer Struktur nicht heruntergehen können. Durch dieses "Fertigsein" in quantitativer Beziehung werden Wachstumsvolgänge ausgelöst. Wăhrend bis dahin jede Zellteilung stets eine Verkleinerung der einzelnen Zellen zur Folge hatte, ein Wachstum des Eies wahrend der Furchung also nicht stattfindet, so muss jetzt jede Tochterzelle erst zur Grösse der Mutterzelle heranwachsen, bevor sie sich ihrerseits teilt. Die Folge davon ist, dass von nun an - d. h. vom Ende des Blastulastadium an - eine Vergrösserung des Keimes stattfindet. Dieses ungleiche - weil nur an einem kleinen Bezirk des Eies erfolgende - Wachstum des Eies bedingt eine Einfaltung - die GastruArchiv f. mikrosk. Anat. Bd. 61. 
lation. ${ }^{1}$ ) Dass der Vorgang der Gastrulation wirklich auf dieses rein mechanische Moment zurückzuführen ist, geht aus dem Verhalten der Lythiumlarven klar hervor. Durch die Einwirkung dieses Salzes wird ja bewirkt, dass kein ungleiches Wachstum der Blastulazellen des Seeigeleies stattfindet. Im Lithium teilen sich alle Zellen gleich schnell, sodass alle Zellen zur gleichen Zeit an die Grenze ihrer Teilbarkeit gelangen. Fangt der Keim jetz an zu wachsen, so findet bei dem gleichmässigen Wachstum der Zellen keinerlei Einfaltung, sondern eine gleichmassige Vergrösserung der Blastula nach allen Seiten hin statt, sodass ein kugelförmiges Gebilde $\mathrm{zu}$ Stande kommt, das fast doppelt so gross ist, wie eine gleichaltrige normale Gastrula.

Ausser diesen Wachstumsvorgängen, wird zu gleicher Zeit an denselben Zellen, die im Blastulastadium die kleinsten des gesamten Keimes sind, noch anderes Differenzierungsgeschehen ausgelöst. Nördlich von der ersten Vrmundeinfaltung legt sich der Embryo bildende Bezirk an, aus dessen mittleren Partien der Kopf entsteht, wälrend die seitlichen das Wachstumszentrum von Rumpf und Schwanz bilden. (Kopsch). Beide Vorgänge - Gastrulation und Herausdifferenzierung des Embryo bildenden Bezirkes - sind völlig unabhangig von einander, wie Figur 8 und Figur 11 lehren. Figur 8. stellt ein Ei dar, das zwar gastruliert hat, an dem eine Differenzierung im Organe aber nicht vor sich gegangen ist, Figur 11 ein $\mathrm{Ei}$, an dem zwar die nervösen Zentralorgane gebildet sind, eine Einstülpung aber nur in sehr beschränktem Maasse ${ }^{2}$ ) stattgefunden hat. (Mir scheint aus diesem Grunde die von Roux gewahlte Bezeichnung "Anentoblastia" treffender für diese Art Missbildungen zu sein

$\left.{ }^{1}\right) \mathrm{R} \circ \mathrm{ux}$ erklärt (44, pag. 342) die Gastrulation in ganz ähnlicher Weise durch ungleiches Wachstum. Nur nimmt $\mathrm{R}$ oux an, dass um diese Stelle eben besonders qualifiziertes Material schon während der Furchung angehäuft worden sei „welches zufolge dieser Qualitäten sowohl am frühesten zu wachsen, wie auch das Medullarrohr mit seinen spezifischen, histologischen und chemischen Qualitäten zu bilden vermag " Ich glaube dagegen, dass dieses erste Wachstum nicht die Folge von an dieser Stelle angehäuften bestimmt qualifizierten Materiales ist, sondern dadurch ausgelöst wird, dass eben an dieser Stelle die Zellen zuerst die Grenze ihrer Teilbarkeit erreichen.

2) Eventuell vielleicht gar keine, weun man annimmt, dass die Kopfdarmhöhle durch Dehiscenz entsteht. 
als die von Hertwig vorgeschlagene „Spina bifida“, weil erstere der Aetiologie derselben - Unterbleiben der Einstülpung mehr Rechnung trägt.) Wir werden also sowohl die Gastrulation als auch die Herausdifferenzierung des embryobildenden Bezirkes als primäre Elementarvorgănge im Sinne von Driesch bezeichnen, Urmund und Embryo bildenden Bezirk aber als primäre Organe. Von diesen primären Organen wird die Bildung sekundärer, von den sekundären die tertiärer Organe ausgelöst u. s. f. Noch einige Worte über den (inneren) Faktor, durch welchen die Herausdifferenzierung des embryobildenden Bezirkes ausgelöst wird. Über seine Natur können wir natürlich nur Vermutungen hegen. Wir werden uns erinnern, dass diese Anlage des Embryo bildenden Bezirkes Hand in Hand mit Wachstumsvorgangen geht, und können dann vielleicht die durch das Wachstum hervorgerufenen chemischen Veriunderungen der Zellen als Reiz auslösende Ursache betrachten. Vom Standpunkte der Weismann'schen Keimplasmalehre aus liesse sich die Sache vielleicht so erklaren: Wahrend der gesamten Furchung findet nur quantitative Teilung der Kerne statt. Wenn nun die Kernmasse soweit verkleinert ist, das eine T'eilung der M a s s e nach nicht mehr möglich ist, so wird dadurch die qualitative Teilung, i. e. die Zerlegung des Keimplasmas eingeleitet. Wie dem auch sei, so viel steht jedenfalls fest, dass dadurch, dass die Zellen an der betreffenden Stelle zuerst die Grenze ihrer Teilbarkeit erreichen, unter die sie nicht herunter gehen können, Wachstums- und Differenzierungsvorgånge an ihnen ausgelöst werden.

Auf dem Boden der von uns mit obigen Zeilen vertretenen Ansicht über die Bildung der primarren Organe wird uns auch das Verständnis der Schultze'schen Doppelmissbildungen leicht werden. Durch die in beiden Zellen stattfindenden Umlagerungen des Dottermateriales werden an dem einen Ei zwei Punkte präformirt, an denen die Zellverkleinerung früher als am Rest des Eies sistiert. Es werden also an $\mathrm{z}$ we i Punkten zugleich primäre Elementarvorgănge ausgelöst. Sehr gut stimmt dazu die Thatsache, dass aus auf dem Zweizellenstadium gedrehten Eiern nur Janusartige Missbildungen entstehen. Duplicitas posterior ist an diesen Eiern noch nie beobachtet worden, wie denn überhaupt eine $\mathrm{r}$ e in e Duplicitas posterior bis jetzt in der gesamten Teratologie der Wirbeltiere meines Wissens 
noch in keinem Falle konstatiert worden ist. Eine solche würde sich allerdings schleht mit unserer Theorie vertragen. Es ist ja sehr wohl denkbar, dass zwei von zwei verschiedenen Bildungszentren ausgehende Individuen $\mathrm{zu}$ einem einzigen mit einander verschmelzen. Die Yersuche von Driesch (14) und Morgan (33) am Seeigelei geben dieser Annahme eine wohl fundierte Grundlage. Es ist aber undenkbar, dass ein einheitliches, ungeteiltes Bildungszentrum zwei Individuen erzeugt. Sehr interessant ist der Vergleich der Morgan' schen und Driesch'schen Verschmelzungsversuche mit den Beobachtungen $\mathrm{zur}$ Strassens. Zur Strassen berichtet von Rieseneibildungen bei Ascaris megalocephala, die dadurch entstanden sind, dass zwei Eier kurz vor oder kurz nach der Befruchtung miteinander verschmolzen (53). Aus solchen Eiern entstanden einfache Embryonen von doppelter Grösse. In den Fallen von Morgan und Driesch aber ist die Verschmelzung offenbar erst im Blastulastadium erfolgt (14, pag. 416). Aus solchen Verschmelzungsprodukten entstanden stets $\mathrm{primär}$ Zwillinge. Es kommt für uns dabei nicht in Betracht, dass durch sekindare Regulationen aus primar doppelten Anlagen oft einfache Embryonen wurden. Der Hauptpunkt ist der, dass aus vor Stabilierung ibrer Struktur verschmolzenen Eiern eillfache Embryonen wurden (zur Strassen), aus nach diesel Stabilierung (infolge Furchung) verschmolzenen aber solche, die wenigstens ihrer primären Anlage nach $Z$ willinge waren. (Driesch, Morgan). Es ist dies ein weiterer nnd wie mir scheint zwingender Beweis gegen die Annahme, dass der zukünftige Embryo irgendwie im Ei präformiert wăle. und füı die Driesch'sche Theorie, dass die Entfaltung der im Kern gelegenen ontogenetischen Möglichkeiten von Reizauslösungen abhängt, die im protoplasmatischen Bau des Eies gelegen sind.

\section{Zasammenfassung und Schluss.}

Fassen wir nunmehr die Ergebnisse unserer Beobachtungen und Überlegungen zusammen:

1. Das unbefruchtete Froschei ist völlig isotrop, sein Bau ist radiăr.

2. Kurz nach der Befruchtung erleidet es durch Schwerkraftswirkung eine Umlagerung seines Dottermateriales, 
durch welches es einen anisotropen, bilateral-symmetrischen Bau erhält.

3. An dem derartig veränderten, befruchteten aber ungefurchten $\mathrm{Ei}$ ist der zukünftige Embryo genau lokalisiert, seine Bilateralität und Polarität festgelegt.

4. Diese Struktur des eben befruchteten Eies kann jedoch immer noch verăndert werden. Sie ist labil. Erst durch die Furchung wird sie stabilisiert. Strukturverändernde Reize können daher nur kurz nach der Befruchtung oder ganz im Beginn der Furchung angreifen, später sind sie wirkungslos.

5. Differenzierungsgeschehen findet beim Froschei während der Furchung nicht statt. Das abgefurchte $\mathrm{Ei}$ ist daher als System genau dasselbe wie das befruchtete, aber ungefurchte Ei. Daher ist das sich furchende Ei nicht im Stande auf aussere Reize in spezitischer Weise zn reagieren, denn zur spezifischen Reaktion gehören speziell differenzierte Organe, die dem abgefurchten Froschkeim noch fèhlen.

6. Die ersten Wachstums- und Differenzierungsvorgănge werden an einer Stelle ansgelöst, an welcher vom Beginn der Furchung an sich die kleinsten Zellen befinden.

7. Als auslösenden Faktor haben wir das Fertigsein der Zellen in quantitativer Beziehung angesehen. Das heisst: Die Grösse der Zellen kann unter eine bestimmte Grenze nicht heruntergehen. Dadurch dass die in der Umgebung des grauen Feldes befindlichen Zellen, aus pag. 377 naher beschriebenen Gründen, diese Grenze vor allen anderen Zellen erreichen, werden zuerst an ihnen Wachstumsvorgănge ausgelöst. Infolge dieses ungleichen Wachstumes kommt es zu einer Einfaltung des Keimes, der Gastrulation. Zur selben Zeit findet an demselben Zellcomplex die Herausdifferenzierung des Embryo bildenden Bezirkes statt, so dass ein kaudaler Zusammenhang zwischen dem Fertigsein der Zellen in quantitativer Beziehung und der Auslösung dieses Differenzierungsgeschehens vermutet werden darf, ohne dass indessen über die spezielle Natur dieses Reiz auslösenden Faktors etwas Naheres ausgesagt werden kann.

8. Die Bildung des Urmundes und die Herausdifferenzierung des Embryo bildenden Bezirkes sind völlig unabhăngig von einander. Diese beiden Vorgănge sind daber als primäre Elementarvorgange im engsten Sinne zu bezeichnen. 
Damit ist also unsere Aufgabe, den Einfluss der Schwerkraft auf die Entwicklung des Froscheies zu analisieren, gelöst. Wir sehen dass von einer nach unbekannten Gesetzen stattfindenden Beherrschung der Furchung und Organbildung durch die Schwerkraft nicht gesprochen werden kann. Der von der Schwerkraft auf die Entwicklung des Froscheies ausgeïbte Reiz muss vielmehr unter die von $\mathrm{Herbs} \mathrm{t}$ in Kategorie II $\gamma^{\prime}$ zusammengefassten strukturellen Reize subsummiert werden. Dass ein Frosch aus dem Froschei entsteht, davon liegen die Ursachen im Kern, dass aber diese potentielle Energie des Kernes in aktuelle verwandelt wird, das liegt an der durch die Schwerkraft geschaffenen Organisation des Eies.

Es ist uns also zum ersten Male gelungen, die gestaltende Wirkung einer normaler Weise angreifenden äusseren Kralt auf die Entwicklung eines Organismus nicht nur zu konstatieren, sondern auch zu analysieren.

Diese Analyse liesse sich vielleicht noch weiter ausdehnen, es könnten noch die feineren Strukturveränderungen speziell der Kerne erforscht werden. Auch die Natur des inneren die Organbildung auslösenden Reizes liesse sich eventuell noch tiefer ergründen. Nie aber kann es uns gelingen zu erfahren, warum denn nun das sich entwickelnde $\mathrm{Ei}$ so und nicht anders aut den betreffenden Reiz reagiert, mit anderen Worten, warum aus dem Froschei ein Frosch wird. Denn das liegt in der Qualität des Eies begründet.

Hier aber ist aller kausalen Forschung ihre Grenze gesetzt. Unsere nur auf Zeit und Raum gehende Vernunft vermag Qualitäten nicht zu ergriinden, sondern nur rein empirisch zu konstatieren. Die kausale Forschung hat sich also nur mit dem Quantitativen zu befassen. Ihre Aufgabe ist gelöst, wenn sie alles Quantitative auf reine Qualität zurückgeführt hat. Diese muss sie als Letztes, Gegebenes hinnehmen. Es geht aus dieser Auffassung hervor, dass die Probleme der kausalen Forschung stets nur rein mechanische sein können. Dabei darf aber nie vergessen werden, dass alles Mechanische, was wir aufzudecken vermögen, sich an Objekten abspielt, die ausser ihrer Quantität, untrennbar mit ihr verbunden, auch Qualitäten besitzen, die etwas prinzipiell anderes, für unsere Vernunft niemals zu Be- 
greifendes sind. Es heisst die Quadratur des Kreises versuchen, wenn man Qualităten auf Quantităten, also etwa auf „Atombewegung", zurückführen will. Qualităten lassen sich nur empirisch konstatieren, aber nicht kausal verstehen. Die vitalistische Schule ist vollständig im Recht, wenn sie dies für unmöglich erklärt. Mechanische Prozesse sind dem Satz vom Grunde unterworfen. Da der Satz vom Grunde die Quintessenz unserer Erkenntnis ist. so können wir bei Kenntnis der Ursache die Wirkung a priori voraussagen. Veränderungen der Qualităt aber lassen sich nicht a priori vorhersagen, sie müssen empirisch ermittelt werden (vergl. 7, pag. 20-23). In einem anderen Punkte aber stehe ich in einem gewissen Gegensatze zu dem Standpunkt, den Driesch in seinen letzten Scbriften einnimmt (12, 13, 16). Ich vermag einen prinzipiellen Unterschied zwischen organischer und anorganischer Qualität nicht zu erblicken. Dass aus einer Eichel ein Eichbaum wird, scheint mir kein grösseres "Wunder" zu sein, als dass ein Lichtstrahl beim Übertritt in ein anderes Hedium num grade so gebrochen wird, dass er in einem Minimum der Zeit zu seinem Ziele gelangt, oder dass zwei Atome Wasserstoff und ein Atom Sauerstoff sich zu Wasser verbinden. Mir scheint eins so wenig "erklïrbar" zu sein, wie das andere. Die Struktur der lebenden Substanz ist offenbar viel komplizierter als die der toten, aber der Unterschied scheint mir kein prinzipieller, sondern nur ein gradueller zu sein (vergl. 43, pag. $264-280$ ). In seinen ersten Schriften hat auch Driescb, wenn ich ihn recht verstehe, diesen Standpunkt eingenommen. Ich muss daher durchaus bestreiten, dass wir das Recht baben, eine eigene, vitale Kausalität zu konstruieren. Ich erblicke in der finalen Betrachtungsweise eine schätzenswerte Bereicherung unserer Methodik, die neben der kausalen Betrachtungsweise uns wertvolle Dienste in del Erkenntnis von Naturgesetzen leisten kann. Aber wir dürfen doch nie vergessen, dass es sich bei Aufstellung des Zweckmässigkeitsprinzip eben um eine subjektive Betrachtungsweise, nicht aber um etwas objektiv Vorhandenes handelt. Drieschs Autonomie der Lebensvorgange involviert aber eine solche objektiv vorhandene $Z$ weckmässigkeit. Er sagt ja selber: „Das Teleologische tritt also hier nicht bloss als formale Betrachtungsweise auf, sondern ganz unmittelbar Spezifitats- im Besonderen ortsbestimmend" (16, 
pag. 179). Es würde den Rahmen dieser Arbeit weit überschreiten, wollte ich mich auf eine Kritik der von Driesch in seinen letzten theoretischen Schriften. $(2,13,16)$ niedergelegten, diesbezüglichen Ansichten einlassen. Aber sollte die Objektivierung einer subjektiven Betrachtungsweise - i. e. der Zweckmässigkeit - nicht auch „auf einer Verwechslung der Verständlichkeit mit der empirischen Wirklichkeit des Geschehens" beruhen, (13, pag. 38)? Ich glaube den Geltungsbereich der finalen Betrachtungsweise nicht besser charakterisieren zu können als mit folgenden Worten Kants. „....., nur wird behauptət, dass die menschliche Vernunft in Befolgung derselben" (i. e. der finalen Betrachtungsweise) „und auf diese Art niemals von dem, was das Spezifische eines Naturzweckes ${ }^{1}$ ) ausmacht, den mindesten Grund, wohl aber andere Erlkenntnisse von Naturgegesetzen wird auffinden können; wobei es als unausgemacht dahingestellt wird, ob nicht in dem uns unbekannten inneren Grunde der Natur selbst die physisch-mechanische und die Zweckverbindung an denselben Dingen in e in em T'rinzip zusammenhängen mögen, nur dass unsere Vernunft sie in einem solchen zu vereinigen nicht im Stande ist, und die Urteilskraft also, als (aus einem subjektiven Grunde) reflektierende, nicht als (einem objektiven Prinzip der Möglichkeit der Dinge an sich zur Folge) bestimmende Urteilskraft, genötigt ist für gewisse Formen in der Natur ein anderes Prinzip als das der Naturmechanismen zum Grunde ihrer Möglichkeit zu denken". (29, § 69).

Freiburg i. B., 7. Juli 1902.

\section{Literaturverzeichnis.}

1. G. B or n, Ueber den Einfluss der Schwerkraft auf das Froschei. Verh. der schles. Gesellsch. f. vaterl. Kultur, April 1884.

2. Derselbe: Ueber den Einfluss der Schwerkraft auf das Froschei. Arch. f. mikr. Anat., Bd. 24, 1884.

2a. Derselbe: Beiträge zur Bastardierung der einheimischen Anuren. Pflügers Arch. f. die ges. Physiol., Bd. 32, 1883.

3. Hans Driesch: Die mathematisch-mechanische Betrachtungsweise morpholog. Probleme der Biologie, Jena 1891, G. F is c b e r.

1) Der gesperrte Druck rührt meistens von mir her. 
4. Dersel be: Entwickl. mech. Studien 1 u. 2. Zeitschr. f. wissenschaftl. Zoologie, Bd. 53, 1891.

5. De rs elbe: Entwickl. mech. Studien 3-6, Zeitschr f. wissenschaftl. Zoologie, Bd. 55 .

6. Derselbe: Entwickl. mech. Studien 7-10, Mitteilungen aus der zool. Stat. zu Neapel 1893.

7. Derselbe: Die Biologie, eine selbständige Grundwissenschaft, Leipzig 1893, W. Engel m a n $\mathrm{n}$.

8. Derselbe: Analytische Theorie der organischen Entwicklung. Leipzig, W. Eng e lma n n, 1894.

9 Derselbe: Die Entwicklung einzelner Ascidienblastomeren, Arch. f. Entwickl. Mech., Bd. 1, 1895,

10. Derselbe: Zur Analyis der Potenzen embryonaler Organzellen. Arch. f. Entwickl. Mech., Bd. 2, 1896.

11. Derselbe: Retrachtungen über die Organisation des Eies und ihre Genese. Arch f. Entwickl. Mech., Bd. 4, 1896.

12. Derselbe: Die Maschinentheorie des Lebens. Ein Wort zur Aufklärung. Biol. Centralblatt, Bd, 16, 1896.

13. Derselbe: Die Lokalisation morphogenetischer Vorgänge. Arch. $f$. Entwickl. IJech., Bd. 8, 1899.

14. Derselbe: Die Verschmelzung der Individualität bei Echinidenkeimen. Arch. f. Entwickl. Nech., 10, 1900.

15. Derselbe: Die isolierten Blastomeren des Echinidenkeimes. Arch. f. Entwickl. Mech., Bd. 10, 1900.

16. Derselbe: Die organischen Regulationen. Leipzig, W.. Engel$\mathrm{m}$ a $\mathrm{n} \mathrm{n}, 1901$.

17. G. Haberlandt: Ueber die Perception des geotropischen Reizes Ber. der Deutsch. bot. Gesellsch., 18. Jahrg.. 1900.

18. Derselbe: Ueber die Statolithenfunktion der Stärkekörner. Ber. der Deutsch. bot. Gesellsch, 20. Jahrg, 1902.

19. Cart Herbst: Experimentelle Untersuchungen über den Einfluss des veränderten chemischen Mediums auf die Entwickl. der Tiere I., Zeitschr. f. wissensch. Zoologie, Bd. 55 .

20. Derselbe: Experim. Untersuchungen etc., 2. Teil, Mitteil. aus der zoolog. Station zu Neapel, II. Band 1895.

21. Derselbe: Experimentelle Untersuchungen etc., 3. Teil, Arch. f. Entwicklungsmechanik, Bd. 2, 1896.

22. Derselbe: Ueber die Bedentung der Reizphysiologie für die kausale Auffrssung von. Vorgängen in der tierischen Ontogenese I., Biolog. Centralblatt, Bd. 141895.

23. Derselbe: Ueber die Bedeutung der Reizphysiologie etc. II, Biolog. Centralblatt, Bd. 15, 1896.

24. Derselbe: Formative Reize in der tierischen Ontogenese, Leipzig, W. Engelman $n, 1901$.

25. Amadeo Herlitzka: Richerche sulla Differenziazione cellulare nello sviluppo embrionale. Arch. f. Entw. Mech., Bd. 6, 1897. 
26. Oscar Hertwig: Ueber den Wert der ersten Furchungszellen für die Organbildung des Embryos. Arch, f. mikr. Anat., Bd. 42, 1893.

27. Derselbe: Ueber den Einfluss niederer Temperaturen auf das Froschei. Arch. f. mikr. Anat, Bd. 51.

28. Versel b e: Beiträge zur experimentellen Morphol. und Entwicklungsgesch. 4. Ueber einige durch Centrifugalkraft in der Entwicklung des Froscheies hervorgerufene Veränderungen. Arch. f. mikrosk. Anat. Bd. $53,1898$.

29. Im m a n u l Ka n t: Die Kritik der Urteilskraft.

30. Fr Kopsch: Experiment. Untersuchungen über den Keimhautrand der Salmoniden. Verh. der Auat. Gesellsch., Berlin, 1896.

31. Derselbe: Gemeinsame Entwicklungsbahnen bei Wirbeltieren und Wirbellosen. Verh. der Anat. Ges. Kiel, 1898.

32. H. L e itg e $\left.b:^{1}\right)$ Studien über die Entwicklung der Farne. Silzungsber. der Acad. der Wissenschaft., Wien, Bd. 80.

33. T. H. Morgan u. Umé Tsuda: The orientation of the frog's egg. Quat. Journ. of micr. sc. vol. 36 nr. 51894.

34. T. H. Morgan: Formation of one Embryo trom two blastulae. Arch. f. Entw. Mech., Bd. 2, 1896.

34a. Derselbe: Half-embryos and whole-embryos from one of the first blastomeres of the frog's egg. Anat. Anz., Bd. 10, 1895.

35. De rselbe: Studies of the "Partial“ Larvae of Sphaerechinus. Arch. f. Entwickl. Mech., 2 Bd., 1896.

36. Derselbe: The number of cells in Larvae from isolated blastomeres of Amphioxus. Arch. f. EntwickI. Mech., Bd. 3, 1896.

37. Max Moszkowski: Ueber die Wirkung der Schwerkraft auf die Entstehung und Erhaltung des bilateral-symmetrischen Aufbaus des Froscheies. Arch. f. mikr. Anat., Bd. 60.

38. Derselbe: Zur Frage des Urmundschlusses bei Rana fusca. Arch. f. mikr. Anat., Bd. 60.

39. B. N ĕ $\mathrm{m}$ ec: Ueber die Art der Wahrnehmung des Schwerkráftreizes bei den Pflanzen, Ber. d. bot. Ges., 18, 1900.

40. E. P fl ü ge $r$ : Ueber den Einfluss der Sebwerkraft auf din Teilung der Zcllen und die Entwicklung des Embryo. Arch. f. die ges. Physiologie, Bd. 31, 1883.

41. Derselbe: Ueber den Einfluss etc. Arch. f. die ges. Physiologie, Bd. 52, 1883.

42. Derselbe: Ueber den Einfluss etc. Arch. f. die ges. Physiologie, Bd. 34,1884 .

43. Heinrich Rickert: Die Grenzen der naturwissenschaftlichen Begriftssbildung, Tübingen und Leipzig, I. C. B. M o h r , 1902.

44. Wilhelm Roux: Gesammelte Abhandlungen, Bd. 2, Leipzig, W. Engelmann, 1895 .

45. OscarSchultze: Ueber die Bedentung der Schwerkraft für die organisatorische Gestaltung, Sitz. Ber. der phys. med. Ges. zu Würzburg, N. F. 28, 1894.

1) Ist mir nicht zugänglich gewesen. 
46. Derselbe: Ueber die Einwirkung niederer Temperaturen auf das Froscbei. Anat. Anz., Bd. 16, 1899.

47. Derselbe: Ueber die Notwendigkeit der freien Entwicklung des Embryo, Arch. f. mikr. Anat., Bd. 55, 1900.

48. Derselbe: Ueber das erste Auftreten der bilateralen Symmetric im Verlauf der Entwicklung, Arch. f. mikr. Anat., Bd. 55, 1900.

49. Derselbe: Zur Frage von der Bedeutung der Schwerkraft für die Entwicklung des thierischen Embryos, Arcb. f. mikr. Anat., Bd. 56, 1900.

50. E. Stahl: Einfluss der Beleuchtungsrichtung auf die Teilung der Equisetumsporen, Ber. der Deutsch. bot. Ges., Bd. 5, 1885.

51. 0. zur Strassen: Ueber die Riesenbildung bei Ascariseiern, Arch. f. Entw. Yech., Bd. 7, 1998.

52. H. V. W ils on: The Closure of the blastopore in the normally placed frog egg. Anat. Anz., Bd. 20.

53. H. Winkler: Ueber den Einfluss äusserer Faktoren auf die Teilung der Eier von Cystosira barbata. Ber. d. Dentsch. bot. Ges. Bd. 18, 1900.

54. L. Kathariner: Weitere Versuche über die Selbstdifferenzierung des Froscheies. Arch. f. Entw.-Mech. Bd. 14. 1902.

55. F. Keibel: Bemerkungen zu Roux's Aufsatz „Das Nichtnötigsein der Schwerkraft für die Entwicklung des Froscheies". Anat. Anz., Bd. 21, 1902.

56. T. H M organ: The Dispensibility of Gravity in the Development of the Toad's Egg. Anat. Anz., Bd. 21, 1902.

57. Wilhelm Roux: Das Nichtnötigsein der Schwerkraft für die Entwicklung des Froscheies. Arch. f. Entwickl-Mech. Bd. 14, 1902.

\section{Erklärung der Abhildung auf Tafel XIX.}

Fig. 1. Morula eines Eies von Rana fusca, das ant dern Vierzellenstadium in Plattencompression gedreht worden und 20 Stunden lang bei $1-2 \mathrm{Grad}$ Wärme gezüchtet worden war. Vom primär dunklen Pol aus gesehen.

Fig. 1a. Dasselbe vom primär hellen Pol aus.

Fig. 2. Morula eines,Eies, das in Plattencompression auf demVierzellenstadinm gedreht und bei 20 Grad Wärme 11 Stunden lang gezïchtet worden ist. Vom dunklen Pol aus.

Fig. 2a. Dasselbe vom bellen Pol.

Fig. 3. Morula eines Eies. das auf dem Zweizellenstadium in Plattencompression gedreht und bei $10 \mathrm{Grad} 11$ Stunden lang gezïcbtet worden ist. Vom primär dunklen $\mathrm{Pol}$ aus.

Fig. 3a. Dasselbe vom primär hellen Pol.

Fig. 4. Embryo mit bellen Medullarwälsten, einem Ei enstammend, das in Pttüger'scher Zwangslage anf dem Zweizellenstadium gedreht, 24 Stunden bei 1-2 Grad, dann unter Befreiung vom Zwange bei ca. 15 Grad gezüchtet worden war. 
390 Max Moszkowski: Schwerkraftswirkung auf' die Entwicklung etc.

Fig. 5. Durchschnitt durch normale Morula von 11 Stunden (bei $15^{\circ}$ )

Fig. 6. Durchschnitt durch ein $\mathrm{Ei}$, das in Pflüger'scher Zwangslage auf dem Zweizellenstadinm gedreht und bei 2 Grad 20 Stunden lang geziichtet worden war.

Fig. 7. Querschuitt durch einen Embryo, mit linkem schwarzen und rechtem grauem Medullarwulst, einem Ei entstammend, das in Pflüger'scher Zwangslage auf dem Zweizellenstadium gedreht und bei $2 \mathrm{Grad}$ 14 Stuuden lang, dann unter Befreiung vom $Z$ wauge bei ca. 15 Grad gezuchtet worden war.

Fig. 8. Auf dem Vierzellenstadium an der oberen Grenze des granen Feldes mit der heissen Nadel angestochenes Ei, 4 Tage nach der Operation.

Fig. 9. Auf dem Achtzellenstadium am seitlichen Rande des grauen Feldes angestochenes $\mathrm{Ei}, 4$ Tage nach der Operation.

Fig. 10. Auf dem Achtzellenstadium seitlich vom grauen Felde angestoclenes Ei. \pm 'Tage nach der Operation.

Fig. 10a. Querschnitt dnrch dieses Ei in der Höhe der Kopfdarmböle.

Fig. 10b. Querschnitt durch disselbe Ei im Anfang des hinteren Drittels der Medullarwülste.

Fig. 10c. Querschnitt durch dasselbe Ei in der Höhe des Defectes.

Fig. 11. Embryo mit Anentoblastia, aus einem Ei, das vor der ersten Furche an der unteren Grenze des grauen Feldes angestochen wurde, 4 Tage nach der Operation.

Fig. 12. Embryo mit Defekt am hinteren Ende ans einem Ei, das vor der ersten Furche an der dem grauen Felde grade gegeniiberliegenden Grenze von Schwarz und Weiss angestochen wurde, 4 Tage nach der Operation. 